\title{
A BIOLOGICALLY INSPIRED NETWORKING MODEL FOR WIRELESS SENSOR NETWORKS
}

\author{
A Thesis \\ by \\ CHARALAMBOS CHARALAMBOUS
}

\begin{abstract}
Submitted to the Office of Graduate Studies of Texas A\&M University in partial fulfillment of the requirements for the degree of MASTER OF SCIENCE
\end{abstract}

December 2009

Major Subject: Electrical Engineering 


\title{
A BIOLOGICALLY INSPIRED NETWORKING MODEL FOR WIRELESS SENSOR NETWORKS
}

\author{
A Thesis \\ by \\ CHARALAMBOS CHARALAMBOUS
}

\begin{abstract}
Submitted to the Office of Graduate Studies of Texas A\&M University in partial fulfillment of the requirements for the degree of

MASTER OF SCIENCE
\end{abstract}

Approved by:

Chair of Committee, Shuguang Cui

Committee Members, Deepa Kundur Volodmyr Nekrashevych

Srinivas Shakkottai

Head of Department, Costas Georghiades

December 2009

Major Subject: Electrical Engineering 


\author{
ABSTRACT \\ A Biologically Inspired Networking Model \\ for Wireless Sensor Networks. (December 2009) \\ Charalambos Charalambous, B.S., The University of Arizona \\ Chair of Advisory Committee: Dr. Shuguang Cui
}

Wireless sensor networks (WSNs) have emerged in strategic applications such as target detection, localization, and tracking in battlefields, where the large-scale nature renders centralized control prohibitive. In addition, the finite batteries in sensor nodes demand energy-aware network control. In this thesis, we propose an energyefficient topology management model inspired by biological inter-cellular signaling schemes. The model allows sensor nodes to cluster around imminent targets in a purely distributed and autonomous fashion. In particular, nodes in the target vicinity collaborate to form clusters based on their relative observation quality values. Subsequently, the clustered sensor nodes compete based on their energy levels until some of them gain active status while the rest remain idle, again according to a distributed algorithm based on biological processes. A final phase of the model has the active cluster members compete until one of them becomes the clusterhead. We examine the behavior of such a model in both finite-size and infinite-size networks. Specifically, we show that the proposed model is inherently stable and achieves superior energy efficiency against reference protocols for networks of finite size. Furthermore, we discuss the behavior of the model in the asymptotic case when the number of nodes goes to infinity. In this setting, we study the average number of cluster members. 
To my parents, Andreas and Ifigenia Charalambous 


\section{ACKNOWLEDGMENTS}

I have the best of memories from my graduate studies at Texas A\&M University. I have made friends and acquaintances from every corner of the world, all walks of life, and different academic backgrounds, all of whom have been influential in my life one way or another.

I am indebted to my advisor, Dr. Shuguang Cui. His support and supervision throughout my graduate studies have been exemplary and phenomenal, while his unquenchable thirst for knowledge is inspiring. In addition, I want to acknowledge and thank my committee members, Dr. Deepa Kundur, Dr. Volodymyr Nekrashevych, and Dr. Srinivas Shakkottai. I would also like to thank Dr. Robin Strickland at The University of Arizona for making my experience as a teaching assistant unforgettable, while I was in Tucson.

I would also like to thank current and past members of the Wireless Networking and Communication Lab, including Tarun Agarwal, Armin Banaei, Pei Li Cai, Jifeng Chen, Long Gao, Chuan Huang, Meng Zeng, Jiaming Qiu, Jing Wang, Peng Wu, Lili Zhang, and Qing Zhou. I thank them for all the discussions that we had, as well as for making our endless hours at the lab enjoyable. I also thank all of my friends at Texas A\&M University, who are too many to mention, for making a small place like College Station a big, worthwhile experience. In addition, I would like to thank all of my friends back home, whom I have been deprived of for a long time.

Most importantly, I thank my family. I thank them for their support and guidance throughout my life. I thank them for all the good and bad times we had, for all the moments we shared; together, or apart. I thank my sisters Anna and Christina, their husbands Kyriacos and Charis, my grandmother Anna and my grandparents Christos and Rigaina who are no longer with us. I also thank the youngest members 
of our family, my nephew Dimos and my niece Ifigenia for brightening our lives with their joyous laughters. Above all, I thank my parents, Andreas and Ifigenia Charalambous. Everything I am, everything I have achieved is because of them. I could not have asked for better role models to follow while I walk through the paths of life. I hope they are as proud of me as I am of them. 


\section{TABLE OF CONTENTS}

CHAPTER

INTRODUCTION . . . . . . . . . . . . . . . . . . . . . . 1

A. Overview of Sensor Networks . . . . . . . . . . . 2

B. Challenges .................. . . . 3

C. Inspiration from Inter-cell Biological Networks . . . . . . 5

D. Contributions . . . . . . . . . . . . . . 6

II INTER-CELL BIOLOGICAL SIGNALING . . . . . . . . . . . 8

A. Juxtacrine Signaling . . . . . . . . . . . . . . . . . . . . . 8

B. Delta-Notch Signaling . . . . . . . . . . . . . . . 9

1. Lateral Induction . . . . . . . . . . . . . . 10

2. Lateral Inhibition . . . . . . . . . . . . . . 10

3. Mathematical Setup of Delta-Notch Signaling . . . . . 13

C. Summary . . . . . . . . . . . . . . . . 13

III BIOLOGICALLY INSPIRED NETWORKING MODEL . . . . 14

A. Model Behavior in the Context of Target Detection . . . . 15

B. Model Assumptions . . . . . . . . . . . . . . . . . . . . . 18

IV ANALYSIS OF THE BIO-INSPIRED NETWORKING MODEL 20

A. Distributed Clustering Algorithm via Lateral Induction . . 20

1. Discrete-time Formulation . . . . . . . . . . . . 21

2. Compact Clustering via Lateral Induction . . . . . . . 22

3. Convergence Speed . . . . . . . . . . . . . . . . . . 23

4. Stability Analysis . . . . . . . . . . . . . . 24

5. Simulation Results . . . . . . . . . . . . 26

a. Setup ................ . . 26

b. Energy Efficiency, Observation Quality, and Compactness . . . . . . . . . . . . . . 29

c. Convergence . . . . . . . . . . . . . 32

B. Inhibition Phase . . . . . . . . . . . . . . . . . 33

C. Clusterhead Election Phase . . . . . . . . . . . . 35

D. Asymptotic Analysis for Networks of Infinite Size . . . . . 36

1. Random Geometric Graphs . . . . . . . . . . . . 37 
2. Transmission Range and Connectivity in RGGs . . . . 37

3. Average Number of Cluster Members . . . . . . . . . 39

E. Summary . . . . . . . . . . . . . . . . . . 41

$\mathrm{V} \quad \mathrm{CONCLUSION} \ldots \ldots \ldots \ldots$

A. Future Work . . . . . . . . . . . . . . 42

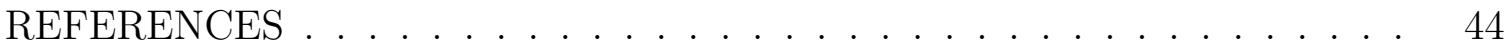

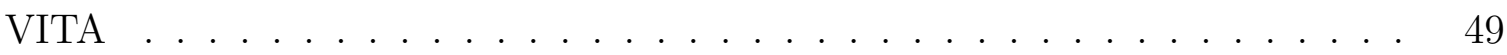




\section{LIST OF FIGURES}

FIGURE

A typical wireless sensor network. . . . . . . . . . . . . . . . . 3

2 The feedback loop in lateral induction for a two-cell system. . . . . . 11

3 The "salt-and-pepper" pattern formed by lateral inhibition. . . . . . 12

4 The three-phase biologically inspired networking model. . . . . . . . 15

5 The timeline of events from deployment to data fusion. . . . . . . . . 17

$6 \quad$ Phase plane for the system of two nodes . . . . . . . . . . . . 25

$7 \quad$ Energy consumption in the clustering algorithm. . . . . . . . . . . . 28

8 Compact clusters via the induction process. . . . . . . . . . . . . 29

9 Average observation quality of cluster members. . . . . . . . . . . 30

$10 \quad$ Energy consumption by induction algorithm. . . . . . . . . . . 31

11 Convergence of the induction algorithm. . . . . . . . . . . . . . 32

12 A typical random geometric graph. . . . . . . . . . . . . 38

13 Average number of cluster members. . . . . . . . . . . . . . . . 39

$14 \quad$ Average number of cluster members for different $\sigma$. . . . . . . . . . 41 


\section{CHAPTER I}

\section{INTRODUCTION}

Wireless communications have sprung into our daily lives and changed the way people and devices communicate. One branch of wireless technology is wireless sensor networks allow devices to connect to each other and collaborate on sensing various physical phenomena ranging from stress on bridges, habitat evolution, traffic patterns, target movement, and so on.

As technology advances, expectations are running high for wireless communications. For sensor networks in particular, the desire for reliable and affordable data collection is enormous, especially given certain critical applications. However, sensor networks have hard energy constraints as each sensor node is equipped with a small battery of finite lifetime. In addition, the large-scale nature of wireless sensor networks, with many consisting of hundreds, thousands, or even millions of sensor nodes, imposes several limitations on coordinating the sensor nodes and controlling the overall network topology. It is clear that sensor network design is challenging given all the above issues.

In this chapter, we first introduce sensor networks and their applications in Section A. We then present some of the challenges and design limitations, thus portraying

the motivation for this thesis in Section B. In Section C we briefly discuss certain properties of inter-cell biological networks, and the lessons that they teach could us in the pursuit of efficient sensor networks. Finally, in Section D we give an overview of the main contributions of this thesis.

The journal model is IEEE Transactions on Automatic Control. 


\section{A. Overview of Sensor Networks}

Recent progresses in wireless communications and electronics have facilitated the development of tiny multi-functional sensor nodes that are low-cost, low-power, and capable of communication in short distances [1]. These tiny sensor nodes consist of components responsible for sensing, data processing, and wireless communications. Their main tasks are to sense physical phenomena, process data, and forward useful information to a fusion center. In particular, the sensing unit is responsible for collecting data from the surrounding environment. The processing unit is in charge of local information processing, such as transforming raw sensed data into a certain digital form via compression or quantization. Finally, the wireless networking unit transmits the locally processed data to a fusion center or a hub node where information from the different sensor nodes is fused to generate the final intelligence. Sensor networks are usually composed of a large number of sensor nodes with a typical structure shown in Fig. 1, where the sensor nodes could forward their data via other intermediate nodes, or relays, to the hub node. Typical applications for wireless sensor networks include, but are not limited to:

- Environment and habitat monitoring: Sensor networks can be deployed to observe various environmental parameters such as humidity or temperature [2], physical phenomena such as forest fires [3] or floods [4], as well as habitat evolution [5].

- Military applications: Sensors can be used for battlefield surveillance, target detection and tracking, and homeland security [6].

- Health monitoring: Sensor networks can provide telemonitoring of human physiological data [7]. 


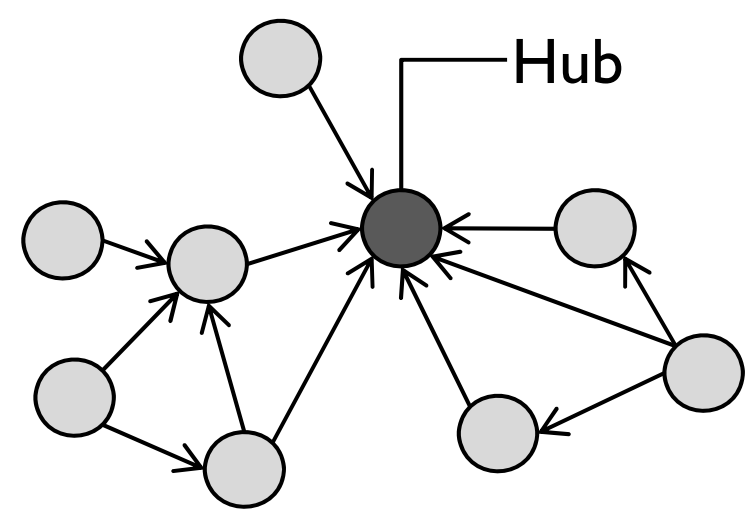

Fig. 1. A typical wireless sensor network.

- Traffic control: Image sensors may be used to monitor traffic conditions at major intersections and highways [8]. An advanced scenario calls for sensors to be attached on the vehicles themselves such that passing vehicles can exchange information such as traffic jam locations [9].

For a more detailed description of sensor network applications please refer to [1] and $[10]$.

\section{B. Challenges}

With the trend towards ubiquitous wireless communications becoming ever-stronger, the large-scale nature of sensor networks demands a high level of self-organization [11], where the participating entities configure themselves into a networking structure that requires minimum central management. As such, sensor nodes need to interact directly with neighboring nodes and constantly react to changing dynamics in their local surroundings.

Such self-organizing systems typically feature flexibility, adaptiveness, robustness, and scalability [11]. The requirement of self-organization in a wireless sensor network (WSN) favors distributed protocols that allow sensor nodes, or clusters of 
sensor nodes, to perform localized sensing and processing $[1,12]$. The absence of a central authority means that the sensor nodes enjoy the sovereignty to decide upon their own destiny, but should also bear the obligation to collaborate with other sensor nodes. In particular, by the cooperative effort a system can achieve better sensitivity and noise immunity via averaging across both space and time [12]. This is the key for success in large-scale sensor networks since each individual sensor node is limited in sensing range, transmit power, and processing capability $[13,14]$.

Among the self-organizing capabilities of sensor networks, autonomous topology control is of paramount importance given its high-level influence over all other aspects of sensor network operations. Many topology control approaches currently available are centralized with inhibitive complexity. This renders them inappropriate for large-scale networks that may operate under hostile conditions where connections to a central controller are rarely guaranteed. Even for the current networking protocols that are optimized for distributed implementations, there exist considerable drawbacks. The most notable one is the scalability issue: As the number of nodes increases, the performance deteriorates at a faster pace. The result is that even the most advanced available ad hoc protocols can only support dozens of nodes. This calls for the design of protocols that could handle wireless sensor networks of perhaps millions of nodes in an efficient manner. The fundamental reason for bad scalability is the lack of distributed autonomy. Many current algorithms, while being designed to be distributed, still possess some central elements in order to maintain a certain level of functionality for the protocol as a whole. The effect of not having a purely distributed protocol, illustrated by the issues of scalability, can be devastating on the practical implementation of a large-scale sensor network.

Among topology control issues, clustering and node scheduling [15, 16, 17, 18, $19,20,21,22,23]$ are two key aspects that directly affect the application-oriented 
network performance. Unfortunately, the current available approaches all bear similar scalability problems as the general topology control for large-scale sensor networks.

\section{Inspiration from Inter-cell Biological Networks}

As we discussed over the issues of current topology control protocols, the existing clustering and duty scheduling algorithms have scalability issues, especially when energy efficiency is one of the design concerns. It remains as a challenging problem how to autonomously form energy-efficient clusters around targets in a large-scale sensor network. Given the unsuccessful history in achieving the above goal, we have to challenge the way that traditional techniques tackle these issues and look for new alternatives [24]. An attractive approach stems from biological research, where researchers point out that living organisms consist of billions of networked cells interacting with each other in a remarkably harmonic way. Comparing the robust biological inter-cell networks with the struggling electronic networks, the contrast is clear: While current sensor network protocols suffer from scalability and efficiency issues, inter-cellular biological networks exhibit purely distributed behavior, stability, high efficiency, and self-healing capabilities. In particular, although in the human body the main activities appear to be controlled by our brain in a centralized manner, it is interesting to note that the development process through which a body grows from several stem cells into a complex structure is solely controlled by distributed mechanisms. More surprisingly, even in the matured body, many activities such as wound healing are controlled by local cell clusters inside the corresponding tissues $[25,26]$.

Therefore, while the design of sensor networks is trying to overcome the issues that we mentioned, the biological inter-cell networks already possess most of the elements that we seek. Specifically, these networks are purely distributed in nature, 
highly efficient, and enjoy autonomous reconfiguration. The following question then arises naturally: Could we design autonomous and distributed large-scale sensor networks by studying and learning from their biological inter-cell counterparts which have been polished by natural selection for millions of years? Thus inspired, we seek to design networking protocols via a methodology motivated by recent biological results, which indicate that billions of cells in organisms autonomously control their growth and interactions in both collaborative and competitive manners.

\section{Contributions}

In this thesis, we first discuss the controlling mechanism behind successful biological networks. We introduce terms such as inter-cell signaling and juxtacrine signaling. The latter is a class of inter-cell signaling wherein cells interact only with cells with which they are in direct contact. We narrow down our discussion to Delta-Notch signaling, a form of juxtacrine signaling that involves the Delta and Notch transmembrane proteins. Given the nature of the feedback among neighboring cells, the Delta-Notch signaling scheme gives rise to two mechanisms: lateral induction and lateral inhibition. In the former, the participating cells adopt the same steady state fate, while in the latter, they adopt different steady states. Thus, certain desired spatiotemporal patterns are possible with such signaling schemes.

Once we understand how biological networks function, and how the cells interact with each other, we translate the inter-cell signaling schemes into an inter-node communication/control model for large-scale wireless sensor networks. The example application that we have in mind is distributed target detection. When a target appears in the field of interest, sensor nodes first collaborate via lateral induction to create a cluster surrounding the target. The nodes comprising the cluster subsequently 
compete via lateral inhibition to achieve active status while the rest remain idle. Active nodes then compete to become the clusterhead. We provide the mathematical formulation for the distributed clustering algorithm that conforms to the biological models. We argue that such a process leads to compact clusters that do not burden the network with distant intra-cluster communications. Moreover, we present the mathematical formulation for the competitive side of the model, namely the process for selecting the active cluster members as well as for electing the clusterhead.

We first focus on networks of finite size, where we provide stability analysis for the proposed clustering algorithm. We compare our model to reference schemes and illustrate its energy efficiency. We then turn our attention to networks of infinite size and examine asymptotic results for our model. We introduce random geometric graphs that offer a good abstraction for a wireless sensor network, and examine the average numbers of cluster members.

This thesis is organized as follows. Chapter II provides biological background that helps us understand the basis of the proposed model. Chapter III formally defines the networking problem that we address. In Chapter IV we describe the proposed network control model based on the inter-cell lateral induction and inhibition mechanisms, with a focus on a biologically inspired clustering algorithm in networks of finite size. We also examine infinite-size networks and certain asymptotic results regarding our model. Finally, in Chapter V we conclude our work and identify open challenges for future research. 


\section{CHAPTER II}

\section{INTER-CELL BIOLOGICAL SIGNALING}

Biologists found that during certain biological tissue development, e.g., in the tissue growth of the chick inner ear, Notch protein [27] first drives multiple cells in the same area to adopt similar characters to form a prosensory patch, and then mediates the hair cell versus supporting cell differentiation within the patch. The first process is controlled by Notch-signaling lateral induction, and the second one by Notch-signaling lateral inhibition [27]. Both are inter-cell signaling schemes that we explain in this chapter, motivating us to develop distributed sensor clustering and node activation control models.

\section{A. Juxtacrine Signaling}

As we discussed before, with the help of what we know about inter-cell biological networks, we may be able to find an efficient and distributed solution for topology control in large-scale sensor networks. In cellular networks, biologists found that during the body development, all the cells are roughly the same at a certain early stage. This is reminiscent to the initial random deployment of sensor nodes. Then, after some kind of interactions among themselves, the cells are grouped into multiple clusters where each cluster evolves into a particular tissue. Furthermore, within each cluster, some cells are determined to be active cells, while the rest become inactive supporting cells. This whole process is controlled by inter-cell signaling schemes.

Inter-cell signaling is essential in the development of biological multi-cell systems. The signals engaged may take various formats and act over a wide range of length or time scales. In particular, inter-cell signaling generally involves the production of 
ligand $^{1}$ by the transmitting cells and its detection by specific receptors expressed by receiving cells, where the ligand is the mediator of the signal [28]. Juxtacrine signaling is a special class of inter-cell signaling, where ligands anchored in the membrane of a cell bind to and activate receptors on the surface of immediately neighboring cells [28]. As a result, signaling within a tissue can only occur among cells that are in direct contact with each other. With suitable feedback among receptor activation and expression levels of ligand, juxtacrine signaling is an efficient mechanism for the long-range propagation of localized signals, and thus the generation of spatiotemporal patterns [28].

\section{B. Delta-Notch Signaling}

A particularly well-documented juxtacrine signaling scheme is the Delta-Notch signaling [27, 28, 29, 30], where both Delta and Notch are transmembrane proteins. Extensive genetic and biochemical studies on the Drosophila fly embryo have identified the product of the neurogenic gene Notch (and its homologues in organisms other than the fly) as the receptor for pattern formation activity, and the product of the neurogenic gene Delta as the corresponding ligand, expressed in the cell delivering the pattern formation mechanism [29].

Therefore, it has been well understood that in the Delta-Notch system, the activation of the Notch pathway (the receptor) by Delta (the ligand) affects the Delta activity of the receiving cell. Since the transmitting cell is also one of the neighboring cells of the receiving cell, the affected Delta activity in the receiving cell will be fed back to the receptors of the transmitting cell, leading to interactive closed-loop dynamics. As such, with the feedback loops being its basic premise, Delta-Notch

${ }^{1}$ Ligand: A small signaling molecule that binds to a protein or receptor. 
signaling between cells is the main controlling scheme for cell pattern generation. Depending on the nature of the Delta-Notch feedback loops among neighboring cells, the pattern-generating processes can be classified into two different categories [27, 28, 31]: lateral induction and lateral inhibition. In the following subsections, we explain the mechanisms behind these two signaling schemes.

\section{Lateral Induction}

Lateral induction is a process by which a cell heading for a particular fate induces its neighbors to adopt the same fate. Specifically, if the Notch activation up-regulates Delta activity in the receiving cell, then this phenomenon propagates to neighboring cells (including the original transmitting cell), stopping only when this externallydriving up-regulation is beaten by internal degradation factors. Eventually a certain region is formed where all the cells in it achieve saturated Delta expressions, corresponding to the formation of a functional patch of cells in the early stage of biological body development. Therefore, the main characteristic of the lateral induction mechanism is a feedback loop that is capable of amplifying initial similarities of membrane levels in a neighborhood of cells, such that a homogeneous spatial pattern is generated. The feedback loop is illustrated in Fig. 2. In this two-cell system, active Notch within one cell indicates the reception of induction from the neighboring cell, which in turn up-regulates Delta activity in the former cell, increasing its ability to deliver induction to its neighbor, and so on. Eventually both of the two cells will adopt the same fate.

\section{Lateral Inhibition}

As a counterpart of the lateral induction mechanism, lateral inhibition is a process by which a cell heading for a particular fate inhibits its neighbors from adopting the 


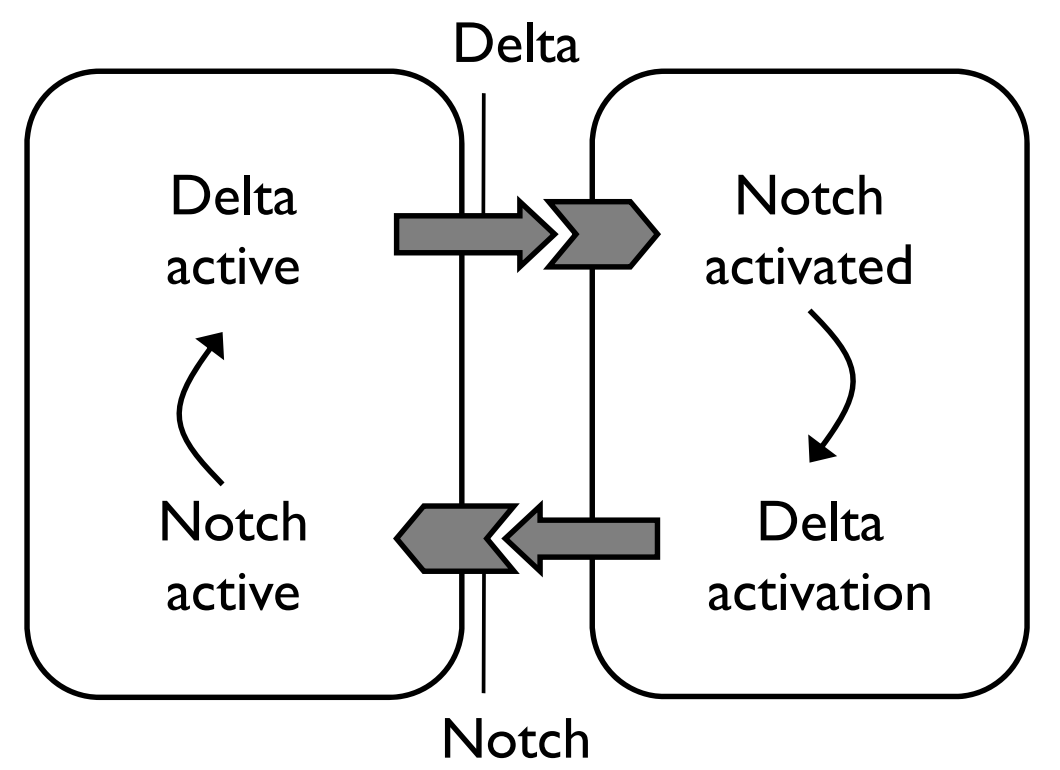

Fig. 2. The feedback loop in lateral induction for a two-cell system. Reception of induction (Notch activation) increases the ability to deliver induction (Delta activity).

same fate. The cells within such a functional patch usually develop further into the differentiation stage, where some cells remain active while the rest are deactivated. For example, during the development of chick inner ear, only a certain portion of sensory hair cells remain active (to grow the hair) within the prosensory patch [27]. Such a differentiation process occurs when Notch activation down-regulates Delta activity in the receiving cell. In turn, the former cell deactivates Notch levels in the neighboring cells, whose Delta activity is in this way up-regulated. This phenomenon propagates to neighboring cells, and eventually a "salt-and-pepper" pattern emerges across the cell lattice where some cells achieve saturated Delta expressions, while others have near-zero Delta expressions. Therefore, the feedback loop present in the lateral inhibition mechanism is capable of amplifying initial differences of the membrane levels in a neighborhood of cells, such that an inhomogeneous spatial pattern is generated. Such an inhomogeneous pattern can be seen in Fig. 3. Cells 


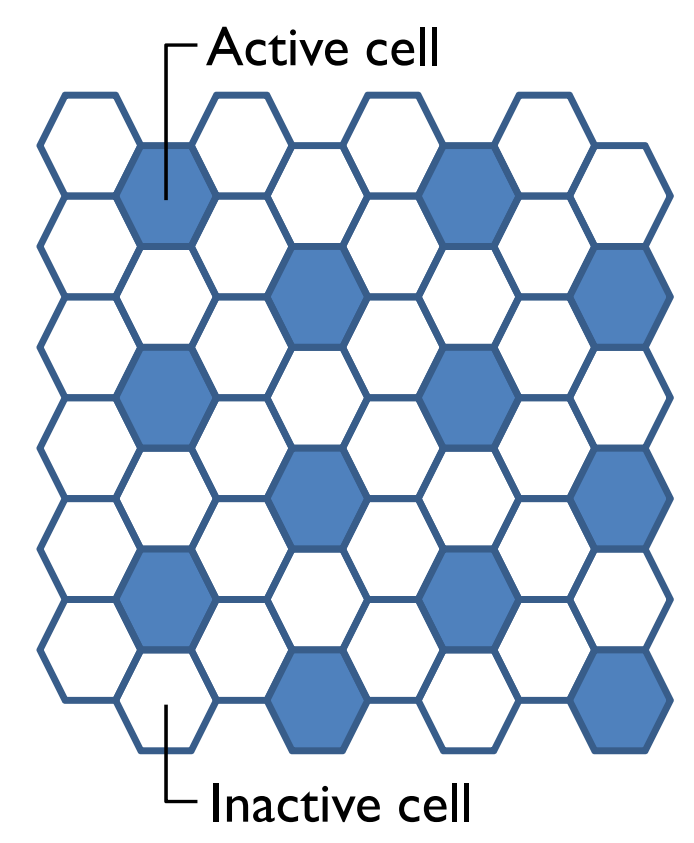

Fig. 3. The "salt-and-pepper" pattern formed by lateral inhibition. Active (primary fate) cells are scattered among inactive (secondary fate) cells.

with high levels of Delta activity and low levels of Notch activation (dark cells) are considered to be the active cells, and correspond to what is called the primary fate in the case of the developing nervous tissue [32]. These cells are scattered among cells with low Delta activity levels and high Notch activation levels (white cells) that are considered to be inactive, corresponding to the secondary fate [32].

One interesting property that arises due to the differentiation in the lateral inhibition process is what we will call as the inhibition property in this thesis. As is apparent in Fig. 3, the steady-state patterns for a cellular network undergoing lateral inhibition follow these two rules:

(i) No two active cells lie next to each other, and

(ii) No inactive cell can be completely surrounded by other inactive cells, i.e., every inactive cell lies next to at least one active cell. 


\section{Mathematical Setup of Delta-Notch Signaling}

Theoretical biologists and mathematicians have successfully modeled the Delta-Notch signaling process by sets of coupled ordinary differential equations (ODEs) [28, 29, 33, 34]. We consider the simple model in [29], where for the $i^{\text {th }}$ cell, $n_{i}$ denotes the levels of Notch activation, and $d_{i}$ denotes the levels of Delta activity. The following set of ODEs governs the behavior of the $i^{\text {th }}$ cell:

$$
\begin{aligned}
& \dot{n}_{i}=f\left(\bar{d}_{i}\right)-n_{i} \\
& \dot{d}_{i}=\mu \cdot\left(g\left(n_{i}\right)-d_{i}\right),
\end{aligned}
$$

where $\mu$ is a positive constant, $\bar{d}_{i}$ represents the average Delta activity across the neighbors of the $i^{\text {th }}$ cell, and $f\left(\bar{d}_{i}\right)$ is a function representing the production rate of the Notch activation in the cell, in response to the increasing amount of Delta activity in neighboring cells. In particular, the function $f\left(\bar{d}_{i}\right)$ models the effect of either lateral induction or lateral inhibition jointly with the function $g\left(n_{i}\right)$. For example, in [29] the related functions for lateral inhibition are given by

$$
f(x)=\frac{x^{k}}{a+x^{k}}, \quad g(x)=\frac{1}{1+b x^{h}},
$$

where $a$ and $b$ are positive constants, $k \geq 1$, and $h \geq 1$. In the following chapters we will show how to apply these models to control the network topology.

\section{Summary}

We have shown that the inter-cell signaling models foster collaboration and competition among interacting cells. Such behavior is reminiscent of the clustering and node activation control in sensor networks. We thus wish to establish a networking model that will feature the behavior of the distributed and efficient biological networks. 


\section{CHAPTER III}

\section{BIOLOGICALLY INSPIRED NETWORKING MODEL}

In Chapter I we introduced wireless sensor networks and the challenges that we face, especially if the networks are large in scale. In Chapter II we turned our attention to their biological counterparts, and described the notion of inter-cell signaling that forms the basic premise of our proposed model. In this chapter, we translate the biological dynamics to guide the design of certain control models in a large-scale sensor network. Particularly, we propose a three-phase networking model in an attempt to mimic the behavior of biological inter-cell networks. Specifically, as shown in Fig. 4, the three phases of our model are:

1. Lateral Induction Phase: During the first phase of the model, the sensor nodes collaborate to construct a functional cluster via lateral induction in a purely distributed fashion.

2. Lateral Inhibition Phase: Once the cluster has been formed, the second phase of the model involves competition among cluster members via lateral inhibition, again in a distributed fashion. The competition winners are the nodes that gain active status, while the remaining cluster members go to the "sleep" or standby mode and may become active themselves in subsequent iterations of the lateral inhibition phase.

3. Clusterhead Election Phase: The last phase of the model is a variant of the lateral inhibition phase, where the active sensor nodes compete until one of them becomes the clusterhead. Once the clusterhead is chosen, it serves as the root of a minimum spanning tree (MST) and gathers data from the rest of the cluster members. 

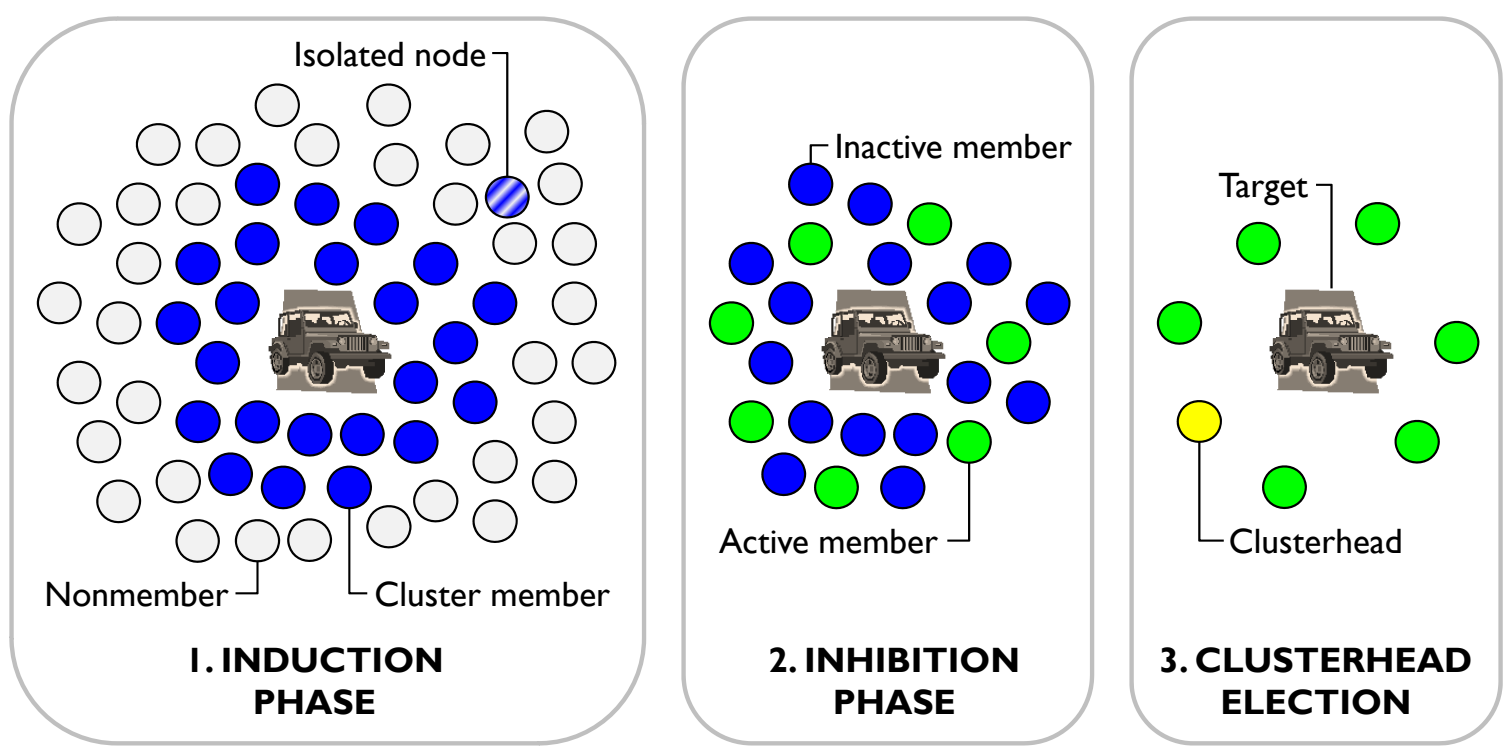

Fig. 4. The three-phase biologically inspired networking model. Phase 1 - Laterally-induced cluster: Sensor nodes with relatively good observation quality values collaborate to form a cluster. Isolated nodes even with good observation quality values are excluded. Phase 2 - Lateral inhibition: Cluster members compete based on remaining energy levels to gain active status. Remaining nodes become inactive cluster members. Phase 3 - Clusterhead election: Active nodes compete based on energy levels and communication cost until one emerges as the clusterhead.

\section{A. Model Behavior in the Context of Target Detection}

One example application that we take on for such a model to be deployed is energyefficient distributed target detection in a wireless sensor network. In the context of target detection, the traditional cluster formation is usually based on the absolute local node observation quality values. However, due to the presence of random noise in the observation field, some scattered nodes that are far away from the target may be included in the cluster, as long as they have good observation quality values. As such, a non-compact cluster may be formed, which is not energy-efficient in terms of data routing at later stages of networking operations. Bearing this in mind, our 
goal is to achieve a compact cluster via the biological lateral induction model in a purely distributed manner. In this way, we aim to prevent isolated nodes, as in Fig. 4, from joining the cluster and burdening the data collection process with distant communications.

As far as the competitive inhibition process is concerned, cluster members decide in a purely distributed manner whether they will be active or not, based on their remaining energy levels. Cluster members with a low energy level compared to their neighbors are considered less "fit" for the task, since they will deplete their energy and die sooner than their more energy-apt counterparts.

In terms of clusterhead election, the competition among active nodes is based on their remaining energy levels, and the overall communication cost as well. The clusterhead should be a node with enough energy level to handle the responsibilities that come with the role, and in a location that does not stress the active set with undesired long hops in the minimum spanning tree.

We next describe the control flow in our networking model. For the target detection application, the control starts when a large number of energy-healthy sensor nodes are randomly deployed into the field of interest. Immediately following their deployment, a preliminary hand-shaking mechanism is enforced such that sensor nodes could determine their neighborhood. Specifically, each sensor node broadcasts a "hello" message and waits for replies from the sensor nodes within its transmission range. Naturally, the number of replies that a sensor node receives indicates the number of its neighbors. Since our model relies heavily on neighbor interactions, we assume that the initial topology constructed is a connected graph, i.e., there exists a communication route (which is usually multi-hop) connecting each sensor node to any other sensor node in the field. In idle status, all sensor nodes are in "sleep" mode in order to conserve energy. During "sleep" mode, functions such as signal processing, 


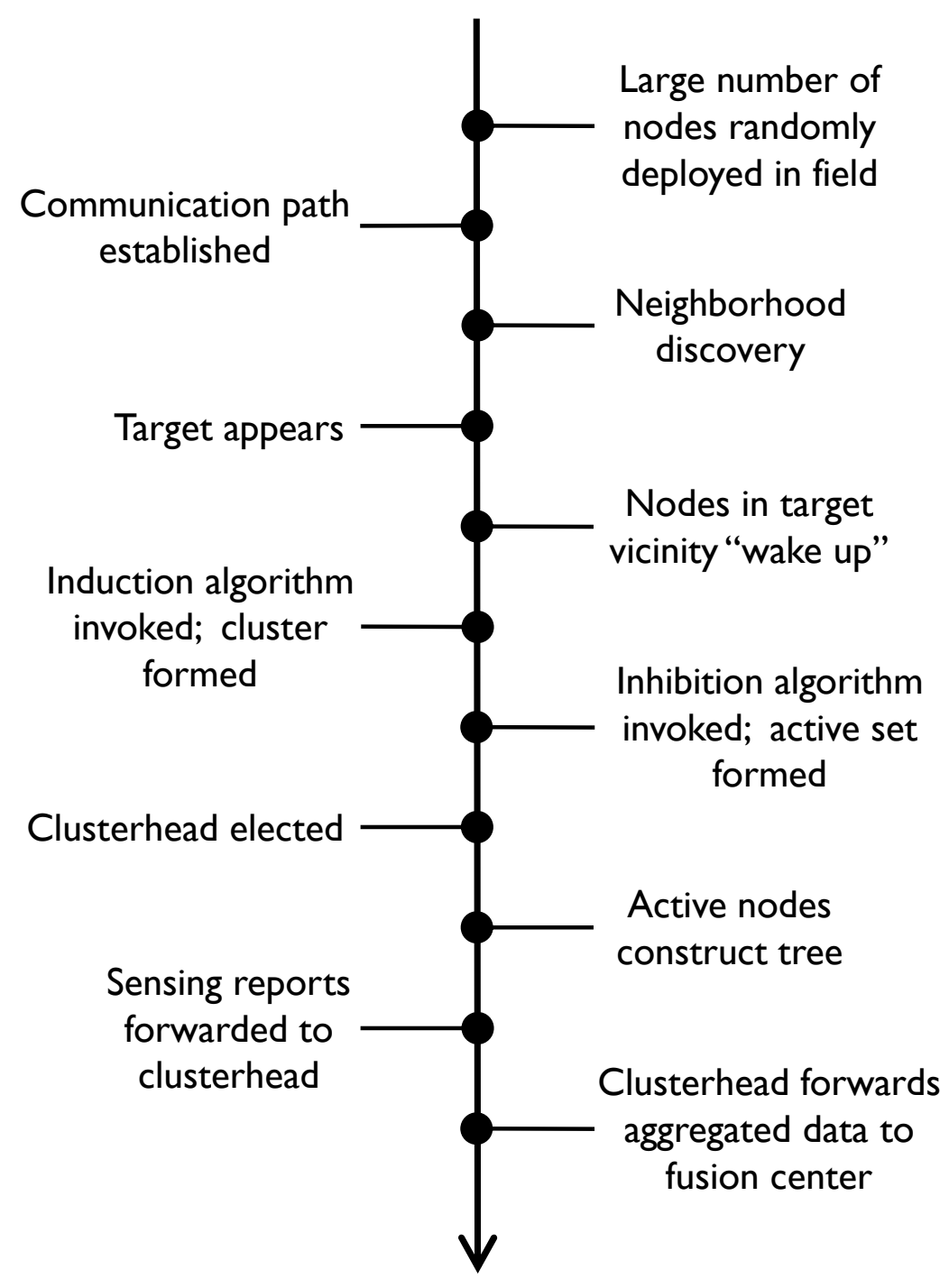

Fig. 5. The timeline of events from deployment to data fusion.

decision-making, and neighbor communications are inactive. However, we assume that the basic sensing unit at each sensor node is constantly active. In the event that a target appears imminent, the sensor nodes whose sensing signals surpass a certain threshold "wake up". At this moment, the clustering mechanism via lateral induction is invoked. The awaken sensor nodes contact their neighbors and exchange the necessary information to run the induction model, and further act according to the evolution of the model parameters. 
Once the cluster is formed, all nodes that are not cluster members go back to their sleeping state. Cluster members by this stage know which of their neighboring nodes are also cluster members and update their appropriate logs and tables accordingly. The cluster members then contact their neighbors and exchange the necessary information to run the inhibition phase. When this phase concludes, only a set of the cluster members is determined to be active. The remaining inactive nodes go back to the idle mode but still retain their cluster membership status, and are eligible to compete in subsequent iterations of the inhibition phase within the same cluster. Such a rotation mechanism is performed such that energy is consumed in a uniform fashion across the entire cluster, by choosing different sets of active nodes across several iterations.

Especially for the phase of clusterhead election, we assume that the active sensor nodes temporarily increase their transmission range by enough proportions so as to be able to communicate with all other active sensor nodes within a single hop. Next, the clusterhead election algorithm is run and the clusterhead is elected. We next assume that the active sensor nodes construct a minimum spanning tree to route their data to the clusterhead. When the clusterhead gathers all the sensing reports from the active nodes, it forwards, after some appropriate processing, the data to a fusion center that is responsible for final data fusion and analysis. The above timeline is illustrated in Fig. 5.

\section{B. Model Assumptions}

The design of inter- and intra-cluster communication protocols, neighborhood discovery, and tree construction are beyond the scope of this thesis, and we therefore assume that their mechanisms are predefined. To conform to the juxtacrine signaling 
models, we also assume that sensor nodes only exchange parameter values with their direct neighbors, thus ensuring that decisions on their fate are performed locally and distributively. Other assumptions we make are that the nodes are immobile and there is a single and stationary target in the network. In addition, the observation signal strength decays smoothly as the nodes are located further away from the target, but the observation quality values may be random due to the random strength of observation noises at different nodes. Moreover, the remaining energy levels of the sensor nodes are not identical across different nodes. 


\section{CHAPTER IV}

\section{ANALYSIS OF THE BIO-INSPIRED NETWORKING MODEL}

In this chapter, we first focus our attention on the clustering process via lateral induction in the context of energy-efficient target detection, as we discussed in the previous chapters. We formally define the induction process mathematically. In addition, we give a discrete-time formulation of the model, which is more suitable for electronic networks that execute algorithms in discrete time. We then perform stability analysis, and we discuss the issue of convergence as well. Afterwards, we provide simulations to verify the clustering algorithm, and examine its energy efficiency. We also study the lateral inhibition process as well as the clusterhead election algorithm. Finally, we conduct certain asymptotic study over the case of infinitely-large networks, where we give an empirical estimate over the average number of cluster members.

\section{A. Distributed Clustering Algorithm via Lateral Induction}

We model the clustering process via lateral induction with a set of two ordinary differential equations for each sensor node, with a system analogous to the biological counterpart in [29]. For the $i^{\text {th }}$ sensor node, let us denote with $q_{i}(t) \in[0,1]$ the time-evolving relative observation quality value of the sensor node in reference to its neighbors, where $\left.q_{i}(t)\right|_{t=0}$ is the absolute observation quality value of the sensor node, reflecting the signal-to-noise ratio (SNR) observed over the target. We assume that the SNR reading of each node has been normalized to be in the range $[0,1]$, according to a predefined maximum SNR reading. Let $s_{i}(t) \in[0,1]$ denote the timeevolving cluster membership indicator, where its steady state value being above a certain threshold means that the sensor node is selected as a cluster member. When

the steady state value is below that threshold, then the sensor node is not selected 
as a cluster member. Specifically, the system of ordinary differential equations for sensor node $i$ is given by

$$
\begin{aligned}
& \dot{q}_{i}=-q_{i}+f_{s}\left(s_{i}\right) \\
& \dot{s}_{i}=\mu \cdot\left(-s_{i}+f_{\bar{q}}\left(\bar{q}_{i}\right)\right),
\end{aligned}
$$

where $\mu$ is a positive constant, and the functions $f_{s}\left(s_{i}\right)$ and $f_{\bar{q}}\left(\bar{q}_{i}\right)$ are in the form of $f(x)$ defined in (2.1), with $a$ and $k$ particularly chosen for each function. The notation $\bar{q}_{i}$ indicates the average relative observation quality value over all the neighbors of the $i^{\text {th }}$ sensor node. In particular, with $\mathcal{N}_{i}$ denoting the set of all the neighbors of sensor node $i$, then

$$
\bar{q}_{i}=\frac{1}{\left|\mathcal{N}_{i}\right|} \sum_{j} q_{j}, \quad \forall j \in \mathcal{N}_{i},
$$

where $\left|\mathcal{N}_{i}\right|$ stands for the cardinality of the set $\mathcal{N}_{i}$.

\section{Discrete-time Formulation}

The system of ODEs shown in (4.1) is a continuous-time model. Since in a sensor network protocols have to be executed in a discrete-time manner, we need to transform the above equations into difference equations. There are numerous methods to transform differential equations to difference equations. Here, we choose the Forward Euler method [35] for the convenience of analysis. According to this method, the derivative $y^{\prime}(t)=f(t, y(t))$ can be approximated as

$$
y^{\prime}(t)=\frac{y(t+T)-y(t)}{T}
$$

with $T$ being the step size. By rearranging parameters appropriately, the above equation becomes

$$
y(t+T)=y(t)+T f(t, y(t)) .
$$


We thus construct an appropriate sequence and index the time by $1,2, \ldots, n, n+1, \ldots$, at integer multiples of $T$, and we then solve for $y$ according to the iterative evolution of the equation

$$
y_{n+1}=y_{n}+T f\left(t_{n}, y_{n}\right),
$$

where $t_{n+1}=t_{n}+T$. We denote by $q_{i, n}$ the relative observation quality value of the $i^{\text {th }}$ sensor node at iteration step $n$, and by $s_{i, n}$ the cluster membership indicator of the $i^{\text {th }}$ node at iteration step $n$. Hence, the equations in (4.1) can be transformed into a set of difference equations for each node:

$$
\begin{aligned}
& q_{i, n+1}=q_{i, n}+T\left\{f_{s}\left(s_{i, n}\right)-q_{i, n}\right\} \\
& s_{i, n+1}=s_{i, n}+T \mu\left\{f_{\bar{q}}\left(\bar{q}_{i, n}\right)-s_{i, n}\right\} .
\end{aligned}
$$

\section{Compact Clustering via Lateral Induction}

The reasoning behind applying induction model to the clustering problem is simple. First, it is highly likely that a sensor node whose neighbors have good observation quality values over the target, has a comparable observation quality value itself. Meanwhile, with energy conservation as one of the main design objectives, it is desirable to have a compact cluster that is energy-efficient in data routing. As such, we want an induction model where a node is more likely to be selected as a cluster member if its neighbors are selected, and vice-versa. In addition, large-scale networks usually operate in noisy environments and thus the distribution of observation quality values across a network is random in nature. As a result, a far-away node might have a much better observation quality value than all its neighbors. With our model, it is likely that this sensor node would be denied cluster membership if its neighbors have a low average observation quality value, such that no far-away isolated sensor nodes are included in the cluster. On the other hand, a node might be selected as a clus- 
ter member even though it might have a mediocre observation quality value, if it is located in a neighborhood of nodes with high observation quality values. Therefore, we see that the collaborative nature of the lateral induction process leads to compact clusters. Such clusters do not burden intra-cluster communications, by denying cluster membership to good, yet isolated, nodes. Meanwhile, they favor compact neighborhoods of good sensor nodes.

Mathematically, the collaborative interaction among sensor nodes that is responsible for the cluster construction is mediated by the average relative observation quality value within the neighborhood of a particular sensor node, since this average value affects its cluster membership indicator value. In turn, a given sensor node also affects the cluster membership indicator values of its neighbors, and the feedback loop continues. For the model in (4.1), by adjusting the system constants $a$ and $k$ in the functions $f_{s}\left(s_{i}\right)$ and $f_{\bar{q}}\left(\bar{q}_{i}\right)$ accordingly, we can change the shape of these functions responsible for the evolution of the induction parameters. In this way, we can control the final cluster size in the steady state in order to fit the needs of a specific sensing application.

\section{Convergence Speed}

In (4.2), the constant $\mu$ represents the ratio between the decay rates of $q$ and $s$, and is therefore a measure of the relative time-scales over which the levels of the relative observation quality and the cluster membership indicator values vary [29]. Increasing the values of $\mu$ and/or $k$ increases the convergence speed [29]; however, extremely large values may lead to numerical issues in discrete implementations, resulting in instability. Actually, the continuous-time biological induction model is inherently stable. In a wireless network though, operations are performed in discrete-time steps by nodes that have finite-bit accuracy. Therefore, selecting extremely large values for 
$\mu$ and/or $k$ might lead to instability caused by numerical issues. Theoretically, the particular choice of $\mu$ does not affect the possible steady states of the system, which are determined by the choices of $a$ and $k$ in the functions $f_{s}\left(s_{i}\right)$ and $f_{\bar{q}}\left(\bar{q}_{i}\right)$. However, when the system admits several equilibrium points, which of the steady states the system settles in is influenced by $\mu$ and initial conditions.

\section{Stability Analysis}

For a system of a large number of nodes, stability issues can only be verified by simulations due to the associated analytical complexity. However, we could gain some insight by studying some small-size systems analytically. We now consider a simple continuous-time system consisting of just two sensor nodes to examine the stability of the induction phase. The equations in (4.1) therefore become:

$$
\begin{aligned}
& \dot{q}_{1}=-q_{1}+f_{s}\left(s_{1}\right), \quad \dot{s_{1}}=\mu\left\{-s_{1}+f_{\bar{q}}\left(\bar{q}_{2}\right)\right\} \\
& \dot{q_{2}}=-q_{2}+f_{s}\left(s_{2}\right), \quad \dot{s_{2}}=\mu\left\{-s_{2}+f_{\bar{q}}\left(\bar{q}_{2}\right)\right\},
\end{aligned}
$$

where the subscripts correspond to sensor nodes 1 and 2. Equilibrium points of the system in (4.3) are given by

$$
\left(q_{1}^{*}, s_{1}^{*}, q_{2}^{*}, s_{2}^{*}\right)=\left(f_{s}\left(s_{1}^{*}\right), s_{1}^{*}, f_{s}\left(s_{2}^{*}\right), s_{2}^{*}\right)
$$

where $s_{1}^{*}$ and $s_{2}^{*}$ are the fixed points of the composite function $\left(f_{\bar{q}} \circ f_{s} \circ f_{\bar{q}} \circ f_{s}\right)$ with $s_{1}^{*}=f_{\bar{q}}\left(f_{s}\left(s_{2}^{*}\right)\right)$ and $s_{2}^{*}=f_{\bar{q}}\left(f_{s}\left(s_{1}^{*}\right)\right)$. For functions $f_{s}$ and $f_{\bar{q}}$ having the form of $f(x)$ in $(2.1)$, there are three equilibrium points with $q_{1}^{*}=q_{2}^{*}$ and $s_{1}^{*}=s_{2}^{*}$. A steady state $x$ is linearly stable if $\left(f_{s} \circ f_{\bar{q}} \circ f_{s} \circ f_{\bar{q}}\right)^{\prime}(x)<1$, but unstable otherwise. Fig. 6 shows the phase plane of the relative observation quality values for this two-node example. The nullclines $q_{1}=\left(f_{s} \circ f_{\bar{q}}\right)\left(q_{2}\right)$ and $q_{2}=\left(f_{s} \circ f_{\bar{q}}\right)\left(q_{1}\right)$ indicate the cases where $\dot{q}_{1}=0$ and $\dot{q}_{2}=0$ respectively. The various trajectories in the phase plane plotted for different 


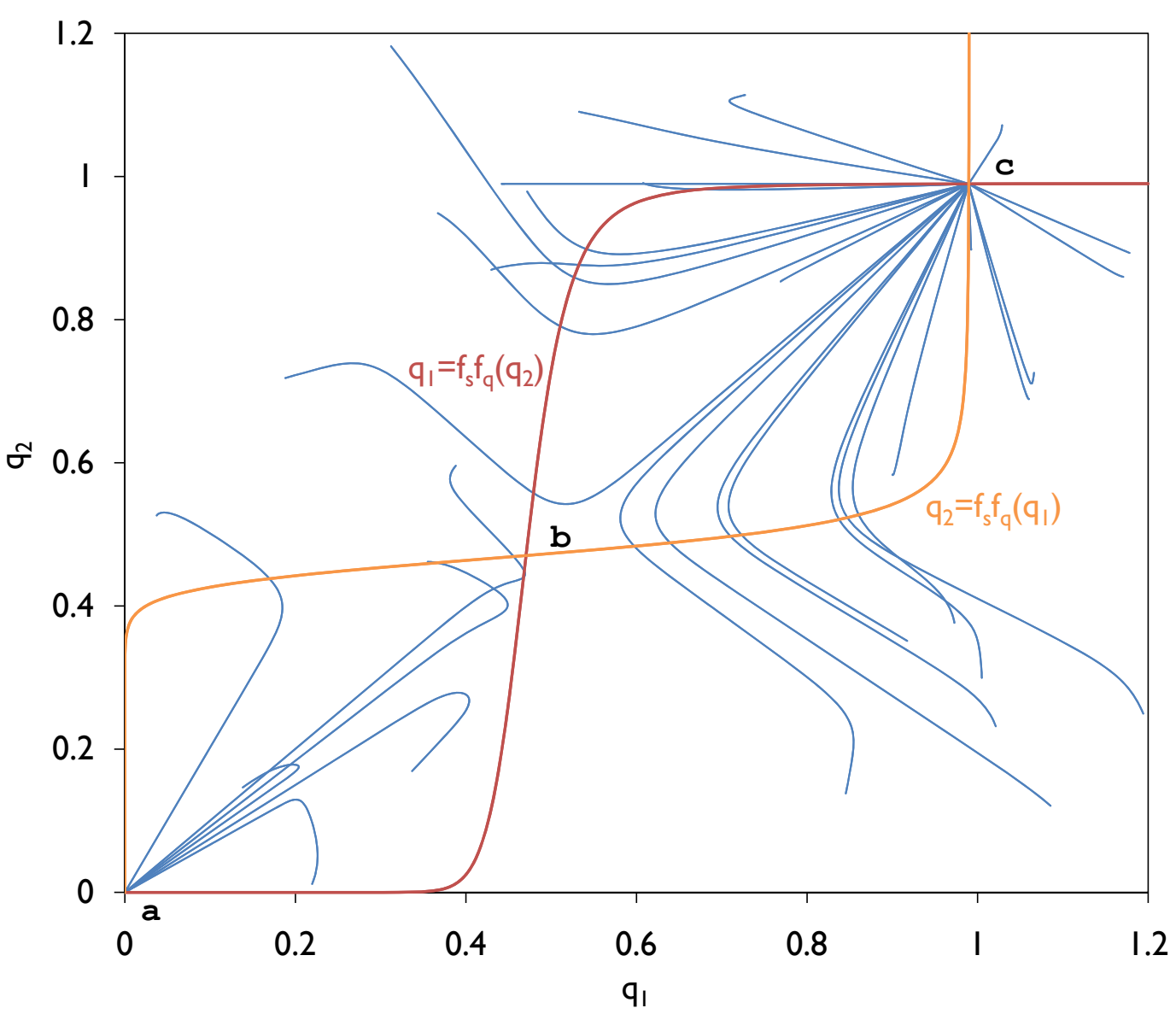

Fig. 6. Phase plane for the system of two nodes. There are three equilibrium points $\{\mathrm{a}, \mathrm{b}, \mathrm{c}\}$, of which point $\mathrm{b}$ is an unstable saddle point, and points $\mathrm{a}$ and $\mathrm{c}$ are stable equilibria. For large values of $\mu$, it is the initial values of $q_{1}$ and $q_{2}$ that determine which of the two stable equilibrium points the system settles in. Here $\mu=10$.

initial conditions of $q_{1}$ and $q_{2}$, show that there is one unstable equilibrium point and two stable equilibrium points. In particular, the equilibrium points a and c are stable, while point $\mathrm{b}$ is an unstable saddle point. As discussed before, the location of the equilibrium points depends on the parameters $a$ and $k$. In this two-node example, $a=0.01$ and $k=3.5$ for the function $f_{s}, a=0.01$ and $k=7.5$ for the function $f_{\bar{q}}$, and $\mu=10$. The three equilibrium points are $\mathrm{a}=(0,0), \mathrm{b}=(0.4705,0.4705)$, and $\mathbf{c}=(1,1)$. 
We next discuss how to achieve one of the two stable equilibrium points in this system. When $\mu \gg 1$, we can make the quasi-steady-state assumption [29] that

$$
f_{\bar{q}}\left(q_{2}\right)-s_{1}=0, \quad f_{\bar{q}}\left(q_{1}\right)-s_{2}=0,
$$

and thus the system in (4.3) reduces to

$$
\dot{q}_{1}=-q_{1}+\left(f_{s} \circ f_{\bar{q}}\right)\left(q_{2}\right), \quad \dot{q}_{2}=-q_{2}+\left(f_{s} \circ f_{\bar{q}}\right)\left(q_{1}\right) .
$$

The phase plane in Fig. 6 shows that when $\mu \gg 1$ it is the initial values of $q_{1}$ and $q_{2}$ that determine which of the two stable homogeneous equilibrium points will be attained eventually. Analogous analysis for $\mu \ll 1$ can be performed, leading to the conclusion that it is the initial values of $s_{1}$ and $s_{2}$ that determine the final steady state (results not shown). Hence, we have chosen a value for $\mu=10 \gg 1$ for the simulations, reflecting our desire to decide upon cluster membership based on the relative observation quality values, i.e., the $q_{i}$ 's.

\section{Simulation Results}

The performance metric by which we assess the clustering algorithm is the total energy consumed by the cluster as a whole to deliver sensing reports to the clusterhead, i.e., the total energy required for each cluster member to forward its sensing report to the clusterhead via a route constructed in the MST.

\section{a. Setup}

We assume that the sensor node with the best observation quality value is chosen to serve as the clusterhead and is therefore the root of the MST constructed by the Kruskal algorithm [36]. The weight function for the Kruskal algorithm is represented by the square of the distance between the cluster members. The initial absolute 
observation quality value of the $i^{\text {th }}$ node depends on its distance from the target, $d_{i, \text { target }}$, as:

$$
q_{i}(0)=\frac{e^{\frac{-d_{i, \text { target }}^{2}}{2 \sigma^{2}}}}{z_{i}},
$$

where $\sigma$ is a deviation measure of the spread of the observation quality distribution. The parameter $z_{i}$ denotes the variance of the observation noise and is assumed to be uniformly distributed with mean 1 between $[1-\alpha, 1+\alpha]$, where $\alpha$ stands for the spread of the noise variance. Essentially, $q_{i}(0)$ represents the normalized SNR at each sensor node and $\left.q_{i}(t)\right|_{t>0}$ represents the time-evolving relative SNR. For such a function, the observation quality values decay exponentially as the square of the distance from the target increases; hence the observation quality function over the field of interest is roughly a "bell-shaped" curve. We assume that $s_{i}(0)=0, \forall i$. The energy required to transmit $b$ bits of data from one node directly to another (single hop) that is located at a distance $d$ away, is defined as:

$$
E_{T X}(k, d)=\epsilon b d^{2},
$$

where $\epsilon$ is the transmission system constant, according to the radio model defined in [16].

We compare our algorithm to a reference scheme loosely based on the initial stage of the Dynamic Convoy Tree-based Collaboration (DCTC) algorithm [37], which creates a tree of nodes surrounding a target as it traverses across the network. The root of the tree is responsible for reconfiguring the tree, by adding or pruning nodes as the target moves. The initial tree construction of the DCTC algorithm is achieved by the nodes that are awake and close to the target.

We simulate in MATLAB for different levels of spread of noise variance, $\alpha$, for a randomly-deployed sensor network over a square field of side length $10 \mathrm{~m}$. There are 


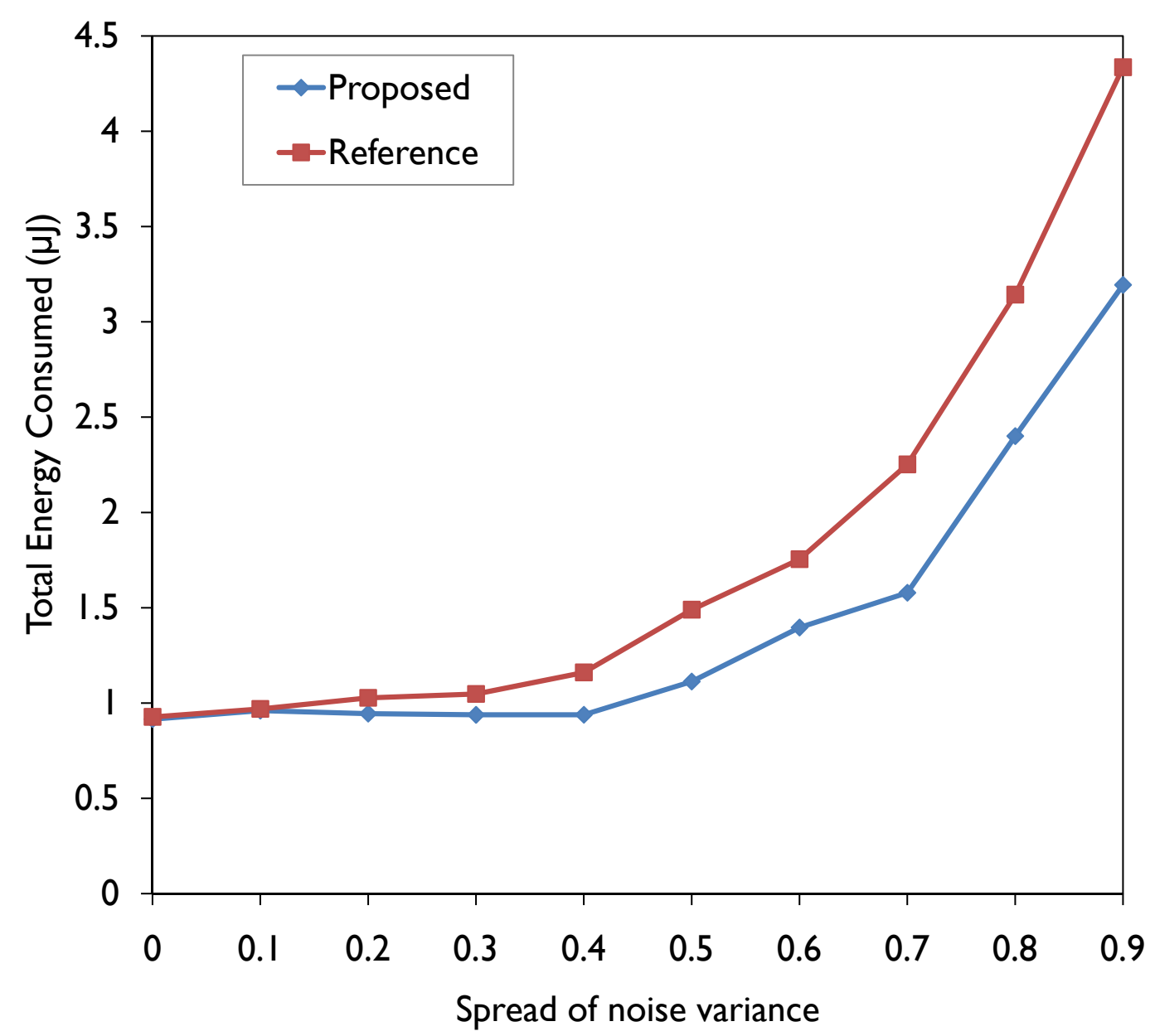

Fig. 7. Energy consumption in the clustering algorithm. Total energy required for all the cluster members to forward their sensing reports to the clusterhead, over the spread of observation noise variance. A compact cluster requires less total energy than one which includes distant nodes.

220 sensor nodes in the field, and each node has a transmission range of $1.5 \mathrm{~m}$. In addition, the target is situated at the center of the field, $\sigma=2$, each sensor node has a 320-bit sensing report to forward to the clusterhead, and $\epsilon=100 \mathrm{pJ} / \mathrm{bit} / \mathrm{m}^{2}$ [16]. We take the average over 200 random network configurations for each of 10 different levels of $\alpha$. We set the threshold for the cluster membership indicator as 0.9. Furthermore, we adopt parallel synchronous updates of the state vectors across the networking sensor nodes, which are done according to the discrete model in (4.2). 


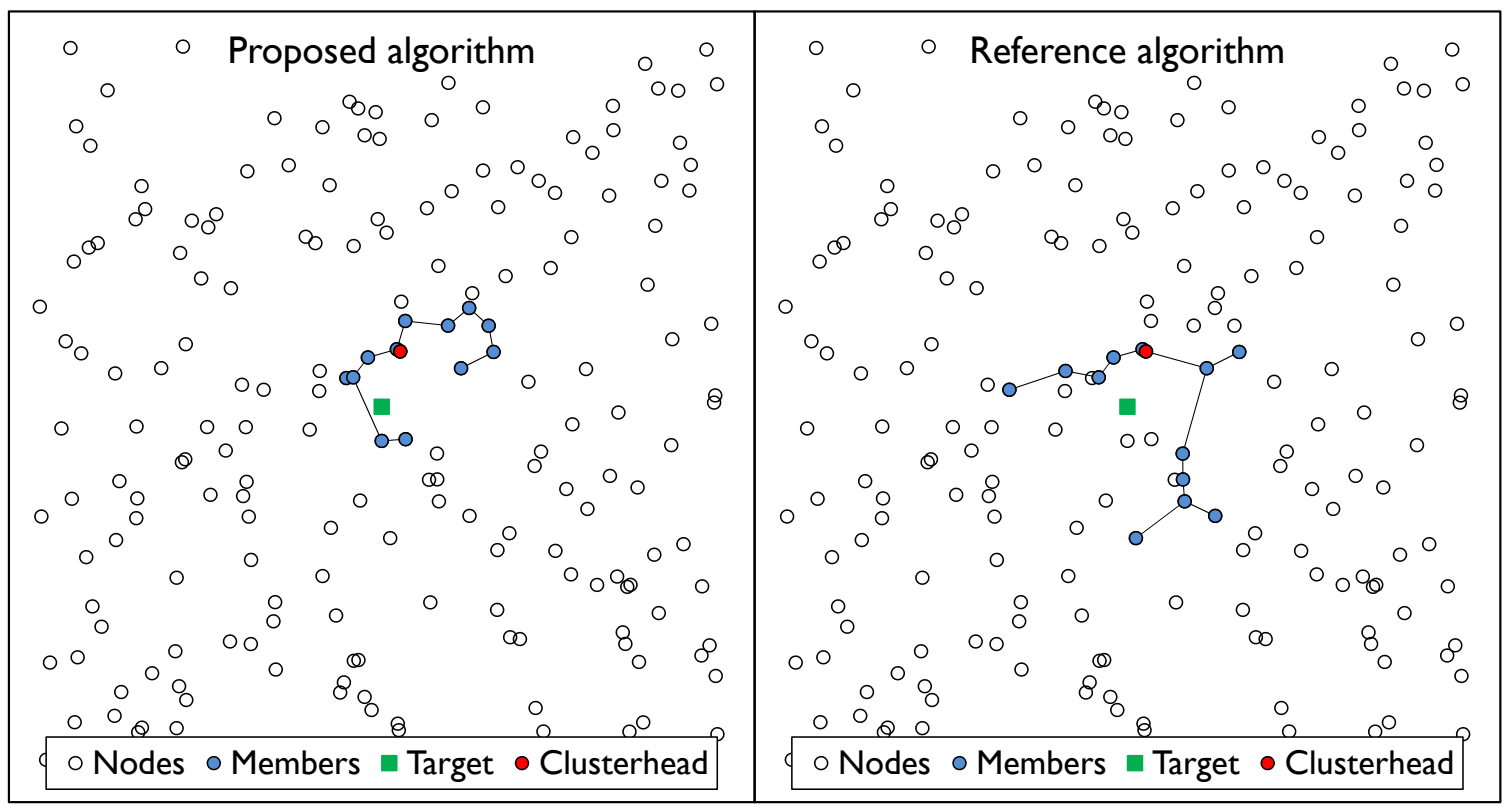

Fig. 8. Compact clusters via the induction process. The proposed algorithm produces a more compact cluster than the reference algorithm. There are 13 cluster members in both cases. Lines connecting nodes stand for the MST routes.

In both schemes, once the cluster members are selected, we assume that all nodes increase their transmission power until they are neighbors with all other cluster members. Then, the MST is formed by the Kruskal algorithm. Once the MST is constructed, the nodes readjust their transmission powers in order to maintain the MST routes with minimum required power. Energy is expended according to the model in (4.6).

\section{b. Energy Efficiency, Observation Quality, and Compactness}

Under low noise spread conditions the two algorithms perform comparably, as shown in Fig. 7. As the spread of the observation noise variance increases, leading to sensor nodes being increasingly misled about their actual distance from the target, the difference in performance becomes clear. Fig. 7 shows that the proposed algorithm expends less overall energy to forward messages from the sensor nodes to the clusterhead for 


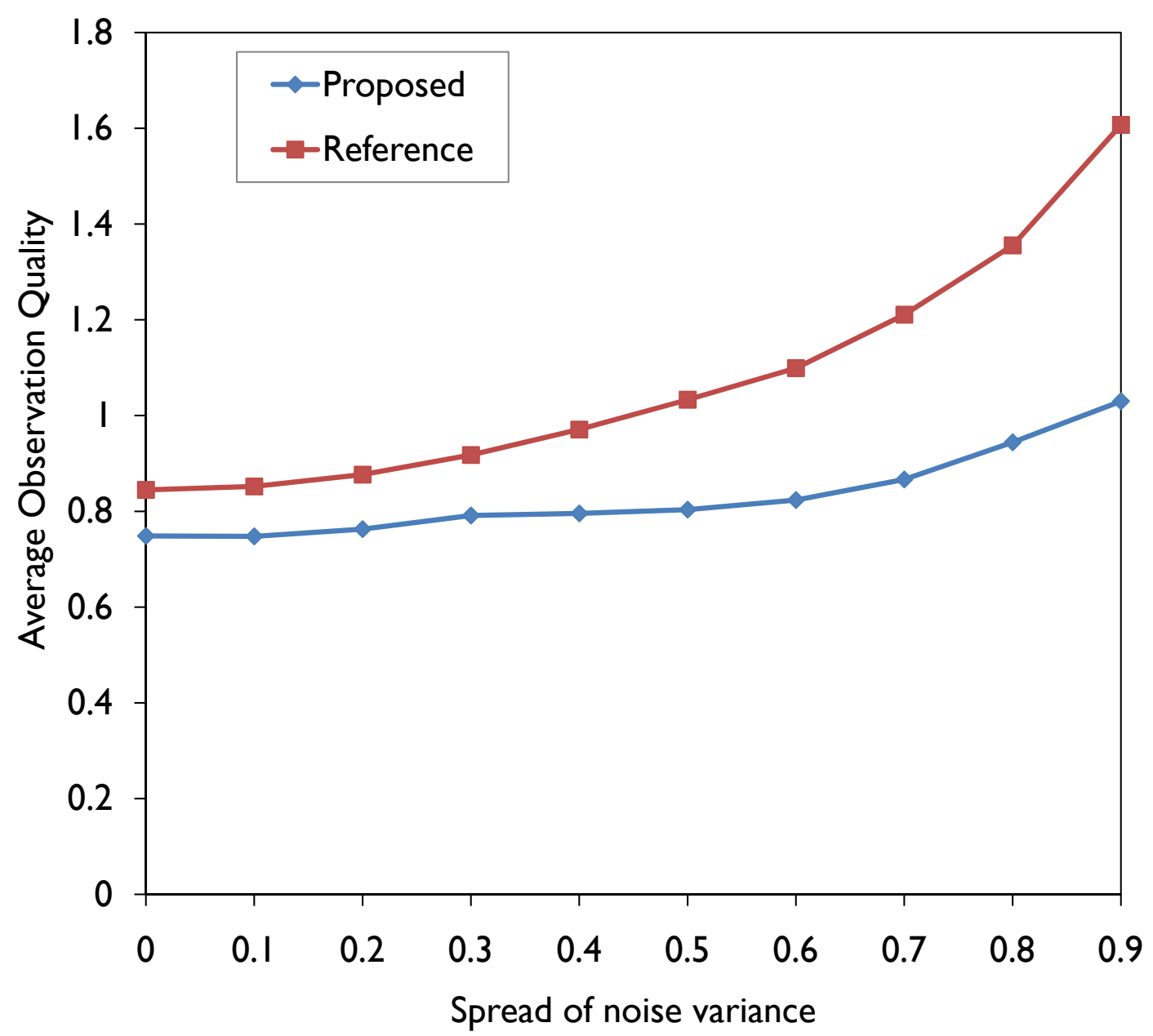

Fig. 9. Average observation quality of cluster members. It is plotted as a function of the spread of observation noise variance. Energy efficiency within a compact cluster comes at a certain expense of observation quality.

the same number of cluster members, especially for relatively large spread of noise variance. This implies that, via inter-node collaboration, the clustering technique via lateral induction constructs a more compact cluster. This attribute can be seen in Fig. 8. This example run of the induction process shows that our proposed model chooses cluster members from sensor nodes that are physically closer to each other, than if the sensor nodes were chosen without any regard towards their surrounding environment. 


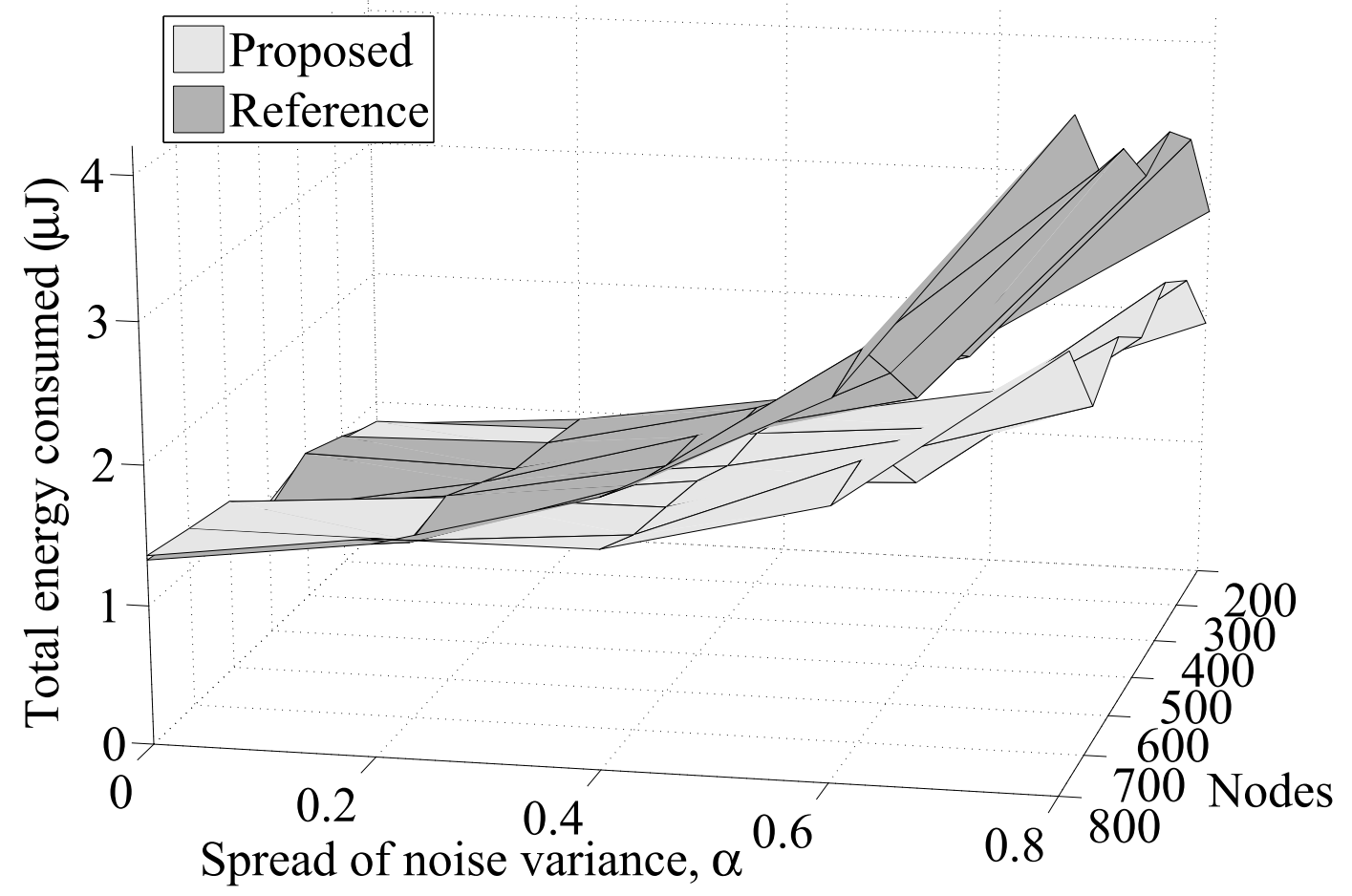

Fig. 10. Energy consumption by induction algorithm. Total energy required for all the cluster members to forward their sensing reports to the clusterhead, as a function of the number of nodes in the network and the spread of observation noise variance.

However, this comes at a certain expense of observation quality. As Fig. 9 shows, for the same number of cluster members, the average observation quality values of the cluster members are lower than those of the reference scheme. This is due to the fact that the lateral induction process might force sensor nodes within a compact neighborhood into joining the cluster as a result of "peer pressure", even though they may not have comparably high observation quality values. At the same time, the induction process might exclude sensor nodes with very good observation quality values from joining the cluster, if they are isolated in a vicinity of nodes with poor observation quality values. As a result, sensor nodes of mediocre observation quality might become cluster members, sacrificing in quality what we gain in energy efficiency. 


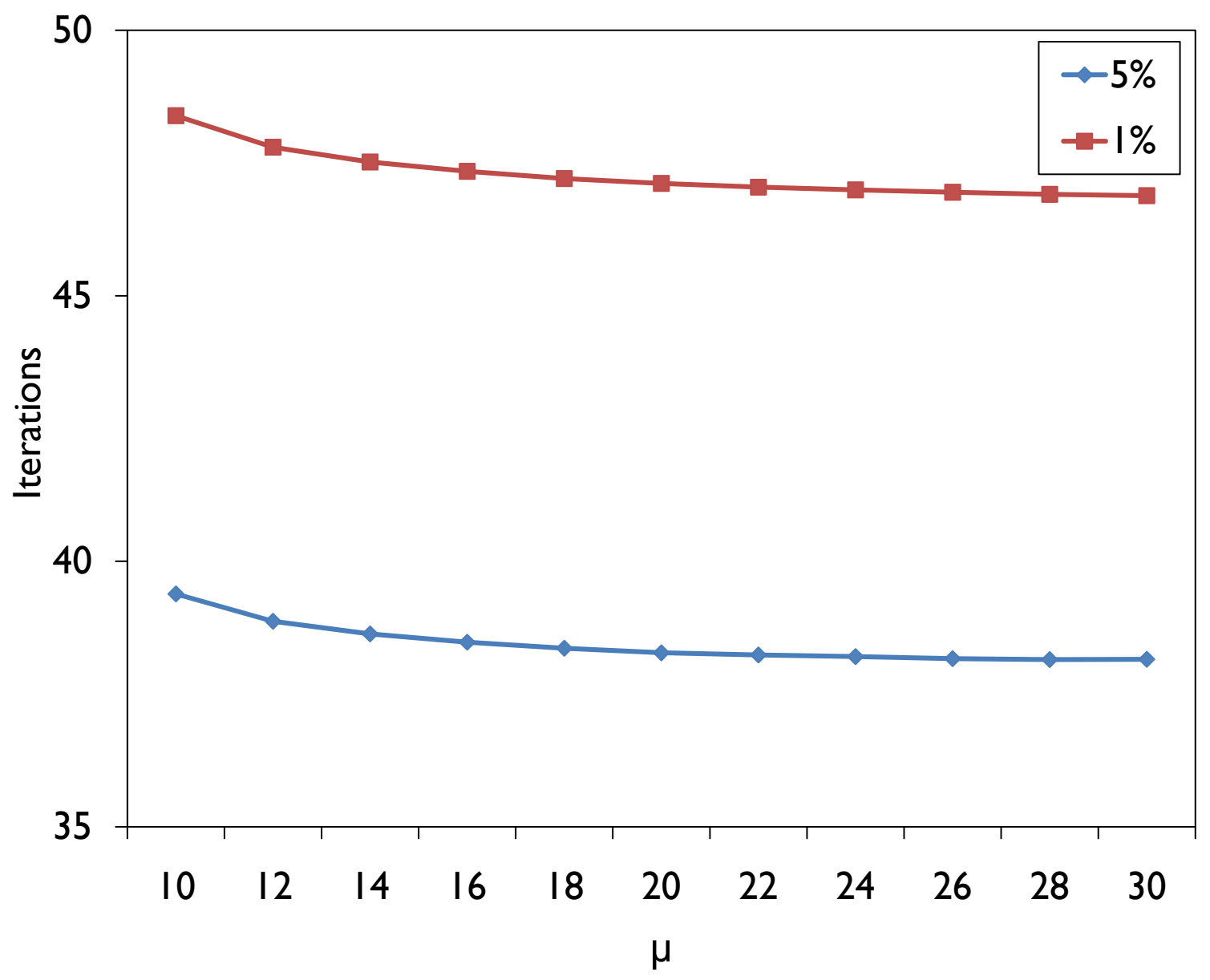

Fig. 11. Convergence of the induction algorithm. Average number of iteration steps needed to settle within $5 \%$ and $1 \%$ of the steady state, versus the value of the constant $\mu$. The system settles faster as $\mu$ increases.

By adjusting our model constants, we could control the tradeoff between the gained energy efficiency and the lost average observation quality. Furthermore, the results depicted in Fig. 10 show that as the network size and the spread of observation noise variance increase, the proposed algorithm improves the relative energy efficiency.

\section{c. Convergence}

In order to examine the effect of the constant $\mu$ on the convergence speed, we simulate a network consisting of 200 sensor nodes, deployed in a square field of side length $10 \mathrm{~m}$. 
Each node has a $1.5 \mathrm{~m}$ transmission range. The step size in (4.2) is $T=0.05$. We average the number of steps necessary for each node to settle within $5 \%$ and $1 \%$ of its final values, over 200 different random network configurations, for different values of $\mu$. Simulations are performed for values of $\mu \geq 10$, so as to conform to the discussion in Section 4. Results in Fig. 11 show that the average number of steps needed to reach the steady state decreases as $\mu$ increases, even though the incremental speed improvement decreases over higher values of $\mu$.

\section{B. Inhibition Phase}

For the differentiation process via lateral inhibition, we have a set of two ODEs for each node that is a cluster member. For the $i^{\text {th }}$ node, we denote with $e_{i}(t) \in[0,1]$ the time-evolving relative remaining energy level of a node with reference to its neighbors, where $\left.e_{i}(t)\right|_{t=0}$ is the absolute remaining energy level of the node. We assume that this reading has been normalized to be in the range $[0,1]$ for each node, according to a maximum energy level. Let $a_{i}(t) \in[0,1]$ denote the time-evolving active status indicator, where steady state values being above a certain threshold mean that the sensor node is selected as an active cluster member, and values being below that threshold indicate that it is not selected. Specifically, the system of ODEs for node $i$ is given by:

$$
\begin{aligned}
& \dot{e}_{i}=-e_{i}+f_{a}\left(a_{i}\right) \\
& \dot{a}_{i}=\nu \cdot\left(-a_{i}+g_{\bar{e}}\left(\bar{e}_{i}\right)\right),
\end{aligned}
$$

where $\nu$ is a positive constant, and the functions $f_{a}\left(a_{i}\right)$ and $g_{\bar{e}}\left(\bar{e}_{i}\right)$ are in the form defined in (2.1), with the constants particularly chosen for each function. The notation $\bar{e}_{i}$ indicates the average relative remaining energy level over all the neighbors of the 
$i^{\text {th }}$ node. As in the induction phase, the continuous-time model can be transformed into a system of difference equations, for which the details are skipped.

Recall that the induction phase has created a compact cluster of "observationquality-fit" sensor nodes. The purpose of the inhibition phase is to choose, among the cluster members, the ones that are most energy-fit in terms of their available energy levels. For example, a node might be suitably located to have a good observation quality value over the target, but might have depleted most of its battery, rendering itself unsuitable for a long active status. As such, we want an inhibition model where a sensor node is more likely to be selected as an active member if its neighbors are not as suitable, energy-wise, to be selected themselves. In addition, we would like for the active nodes to be uniformly spread out across the entire cluster, to maintain a smooth average observation quality when switching between different active sets over time. Indeed, with an inhibition setup, not all the resulting active nodes will be the ones with the most remaining energy levels in the entire cluster, but the ones with remaining energy levels that are comparably high in their own neighborhood. Hence, active nodes will be distributed throughout the whole cluster, in the "saltand-pepper" pattern already observed in biological systems as we discussed before.

Mathematically, the competitive interaction among sensor nodes that is responsible for the active set formation is mediated by the average relative remaining energy level within the neighborhood of a particular node. This average level affects the active status indicator value of that node. In turn, the node affects the active status indicator values of its neighbors, and the feedback loop continues. As in the induction phase, the constant $\nu$ represents the ratio between the decay rates of $e$ and $a$, and is therefore a relative measure of the convergence speed of the inhibition process. 


\section{Clusterhead Election Phase}

The model for the clusterhead election phase is a variant of the lateral inhibition model, with nodes competing to become the clusterhead, which is usually needed within an active set in a given cluster. We model the clusterhead election process via lateral inhibition with a set of three ODEs for each sensor node that is an active cluster member. The first ODE involves $e_{i}(t)$, defined in the same way as in the previous section. Second, let $d_{i}(t) \in[0,1]$ denote the time-evolving distance indicator, defined as follows:

$$
d_{i}=1-\frac{\sum \operatorname{dist}_{i, \mathcal{N}_{i}}}{\max _{i} \sum \operatorname{dist}_{i, \mathcal{N}_{i}}}
$$

where $\sum$ dist $_{i, \mathcal{N}_{i}}$ is the sum of the square of the distance from sensor node $i$ to each of its neighbors, which in this case are all active cluster members, given the one-hop coverage assumption made earlier. In other words, each sensor node $i$ sums up the square of the distance from every other sensor node in the active set, and normalizes it by dividing with the maximum such quantity. The square of the distance is used since we assume that the energy required for node communication is proportional to distance squared, as in (4.6). Thus, a low value of $d_{i}$ means that the node is not suitably located to serve as the clusterhead since it will incur a higher communication cost. Finally, let $c_{i}(t) \in[0,1]$ be the time-evolving clusterhead indicator, where a steady state value above a certain threshold means that the sensor node is selected to be the clusterhead, and a value below that threshold indicates that it is not selected. Due to the fact that such a kind of inhibition takes place on a complete graph comprising all active cluster members, only one node will emerge victorious from this competition since the inhibition property holds. Specifically, the system of ODEs for 
node $i$ is given by

$$
\begin{aligned}
& \dot{e}_{i}=-e_{i}+f_{c e}\left(c_{i}\right) \\
& \dot{d}_{i}=-d_{i}+f_{d e}\left(c_{i}\right) \\
& \dot{c}_{i}=\xi \cdot\left(-c_{i}+w_{e} g_{\bar{e}}\left(\bar{e}_{i}\right)+w_{d} g_{\bar{d}}\left(\bar{d}_{i}\right)\right),
\end{aligned}
$$

where $\xi$ is a positive constant, and $w_{e}$ and $w_{d}$ are nonnegative constants that represent weights on the energy and distance factors, respectively. Moreover, $w_{e}+w_{d}=1$. The functions $f_{c e}\left(c_{i}\right), f_{d e}\left(d_{i}\right), g_{\bar{e}}\left(\bar{e}_{i}\right)$, and $g_{\bar{d}}\left(\bar{d}_{i}\right)$ are in the form defined in (2.1), with the constants particularly chosen for each function. The notations $\bar{e}_{i}$ and $\bar{d}_{i}$ indicate the average relative remaining energy level and average relative distance indicator value over all the neighbors of the $i^{\text {th }}$ node, respectively. As in the induction and inhibition phases, the continuous-time model can be transformed into a system of difference equations, while the constant $\xi$ is a measure of the convergence speed of the clusterhead election phase, with details skipped here.

\section{Asymptotic Analysis for Networks of Infinite Size}

So far in this chapter, we described and studied the clustering model based on lateral induction, the node activation model based on lateral inhibition, and the clusterhead election model also based on lateral inhibition. For finite-size networks, the above models can be directly applied and the performance can be verified by simulations. However, for networks of super-large size, it is hard to verify the performance by simulations. In this section, we attempt to look at asymptotically large wireless sensor networks, and study the asymptotic behavior of the proposed models, with a focus on the induction case. Specifically, we provide estimates for the average value of the cluster size after the completion of the induction phase. 


\section{Random Geometric Graphs}

Before we get into the details, we introduce some notation and definitions. We let $n$ denote the number of nodes initially present in the field, i.e., the number of nodes deployed in the area of interest. Each sensor node has a transmission range of $r$ meters and we assume that it can successfully communicate with every node that lies within its transmission range. We model our network as a random geometric graph (RGG) [38], denoted by $\mathcal{G}(n, r)$.

An RGG is mathematically defined over a metric space $\mathcal{S}$ and a distance measure $\delta$. Let $V$ denote the node set, with $n=|V|$ being the cardinality of the set $V$, i.e., the number of nodes in the metric space. The nodes are randomly deployed in $\mathcal{S}$. The edge set $E$ defines connections between nodes, given as

$$
E=\{(u, v) \mid(u, v \in V) \wedge(0<\delta(u, v) \leq r)\}
$$

In other words, two nodes $u$ and $v$ are connected by an edge if and only if the distance between them (according to the distance measure $\delta$ ) is less than the parameter $r$.

In our WSN scenario we let $S=[0,1]^{2}$, that is, our metric space is the unit square in the two-dimensional Euclidean space. The nodes are uniformly and randomly deployed over the unit square. Thus, for sufficiently large $n$, we also associate a node density to our RGG, which is also equal to $n$. The distance measure used to determine the edge set is the Euclidean distance between two nodes. An example of a random geometric graph consisting of 100 nodes is shown in Fig. 12.

\section{Transmission Range and Connectivity in RGGs}

One of the most prominent properties of RGGs is connectivity. We are usually interested in the transmission range of the nodes such that the WSN is connected with 


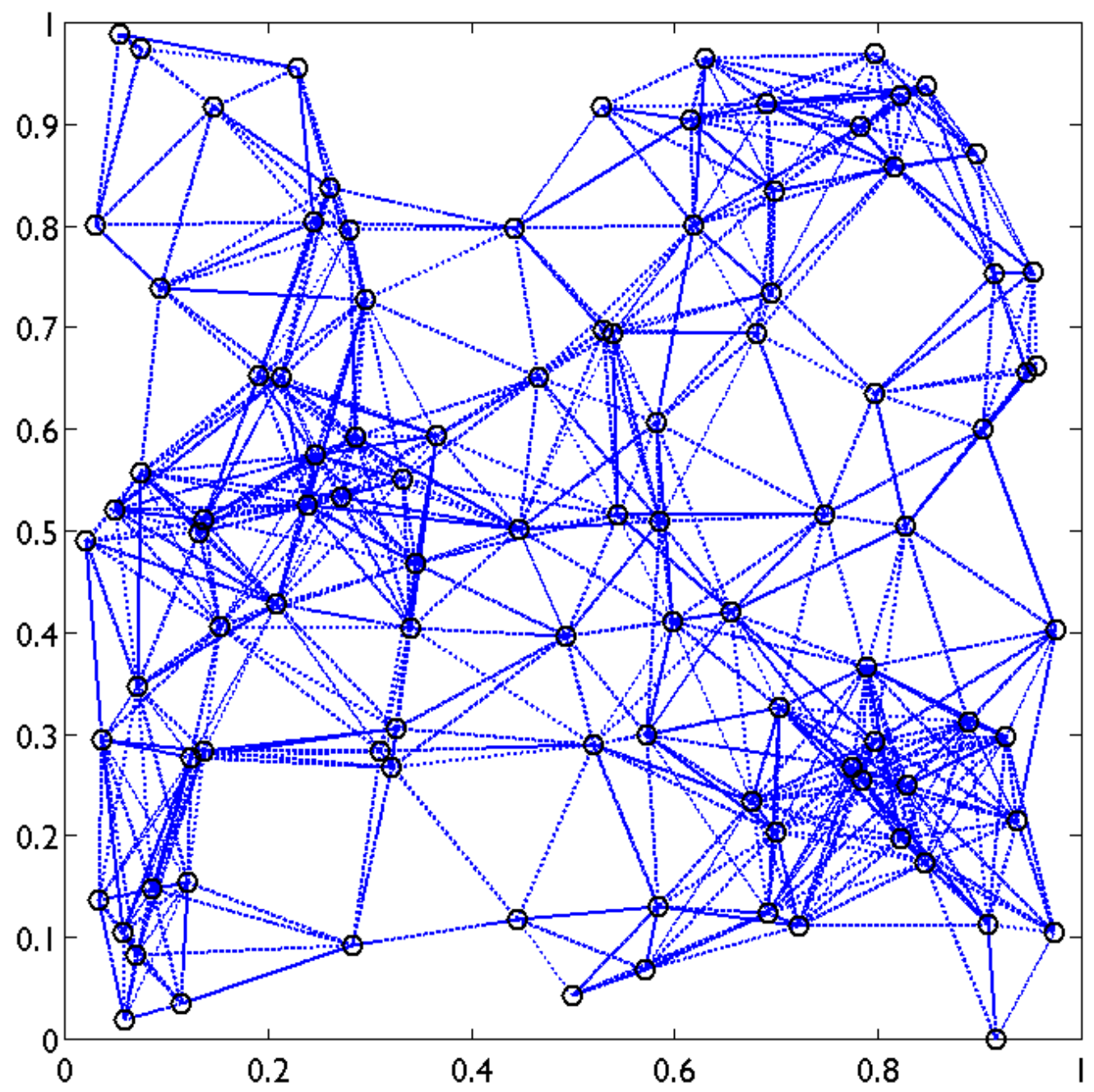

Fig. 12. A typical random geometric graph. The metric space is the unit square, $n=100$, and the transmission radius conforms to $(4.11)$.

high probability (whp). The transmission range $r$ is now a function of the number of nodes $n ; r(n)$. As the number of nodes $n$ present in the network goes to infinity, there exists a critical transmission range $r_{c}$ above which the RGG graph $\mathcal{G}(n, r(n))$ nodes is connected whp. Specifically, the critical radius for connectivity [38, 39] is

$$
r_{c}=\sqrt{\frac{\log (n)}{n}},
$$




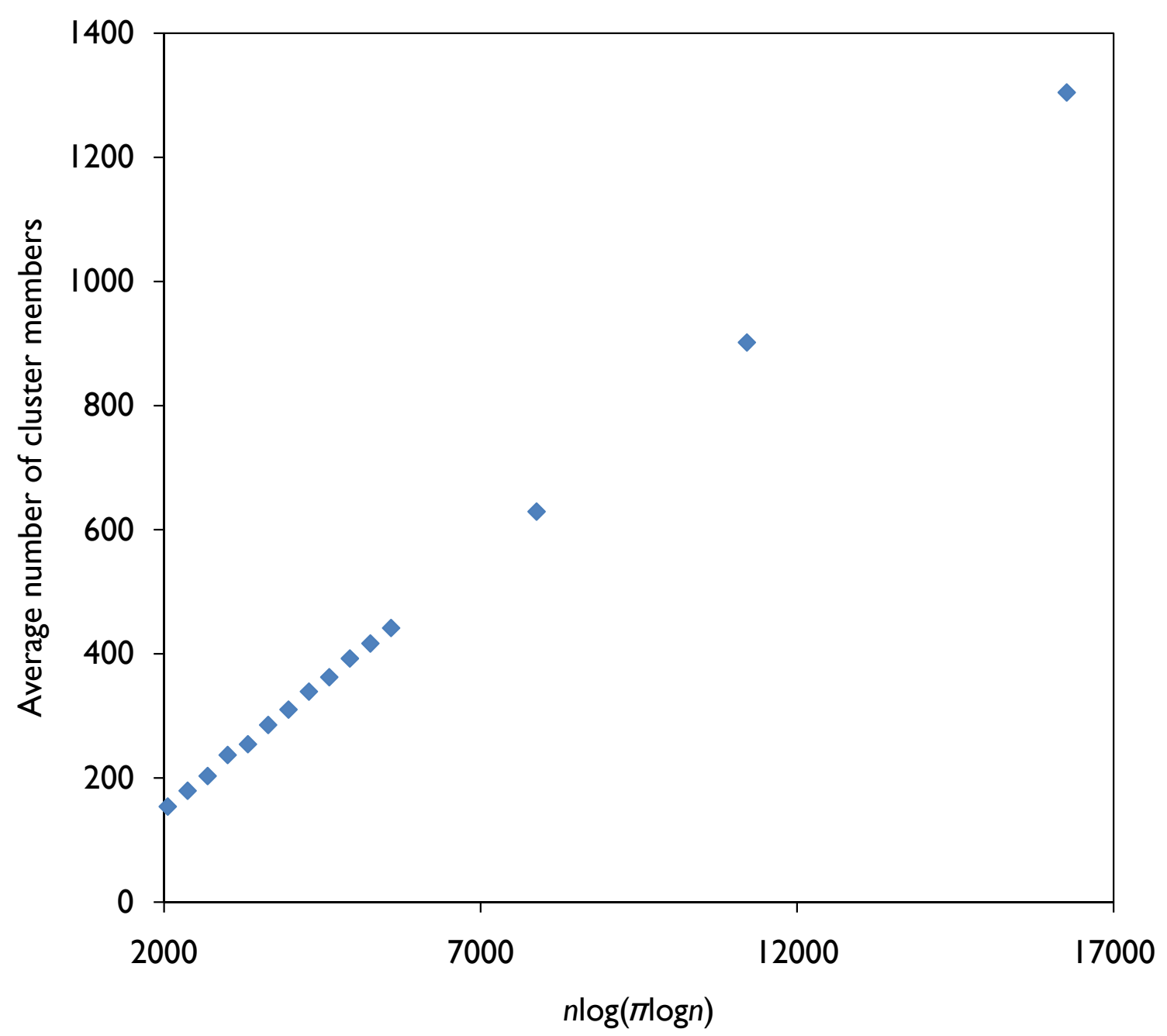

Fig. 13. Average number of cluster members.

where $\log (n)$ is the natural $\log$ arithm. In the asymptotic case where $n$ goes to infinity, an RGG with a transmission range according to (4.11) is connected whp. That is, $\lim _{n \rightarrow \infty} P\left(\left\{\mathcal{G}\left(r_{c}(n), n\right)\right.\right.$ connected $\left.\}\right) \rightarrow 1$.

\section{Average Number of Cluster Members}

We denote by $M$ the set of cluster members after the completion of the induction phase. Let $m=|M|$ be the cluster size, and $\bar{m}$ be the average value of $m$. We are interested in estimating the average number of cluster members, when the observation 
quality function has the form as defined in (4.5). Given a RGG consisting of $n$ sensor nodes, i.e., $\mathcal{G}(n, r)$, and an induction evolution given by (4.1), an empirical estimate of the average number of cluster members after the completion of the induction phase is given as:

$$
\bar{m}=\alpha n \log (\pi \log n)+\beta,
$$

where the term $\pi \log n$ is the average node degree, as can be shown for the given RGG. The terms $\alpha$ and $\beta$ depend on $\sigma$, as well as the thresholds of the switching function in the induction algorithm. The above result can be verified by the following numerical simulations.

For different random realizations, we assume that all networks are deployed over the unit square. The transmission range of each node is as defined for critical connectivity in (4.11). The target to be detected is situated at the center of the field, namely at coordinates $(0.5,0.5)$. We set the threshold for the cluster membership indicator as 0.5. We adopt parallel synchronous updates of the state vectors across the networking nodes. Fig. 13 shows the simulated average cluster size simulations for RGGs of size up to $n=5000$, where each point is average over 500 random runs. We see that there exists an affine relationship between the average number of cluster members and the quantity $n \log (\pi \log n)$. As the number of nodes in the network increases, the number of cluster members increases linearly as well.

Fig. 14 shows the average number of cluster members over network size, for different values of the constant parameter $\sigma$ in the observation quality function as defined in (4.5). As expected, larger values of $\sigma$ mean that the observation quality values decay more slowly with distance, and hence more sensor nodes have the opportunity to become cluster members. 


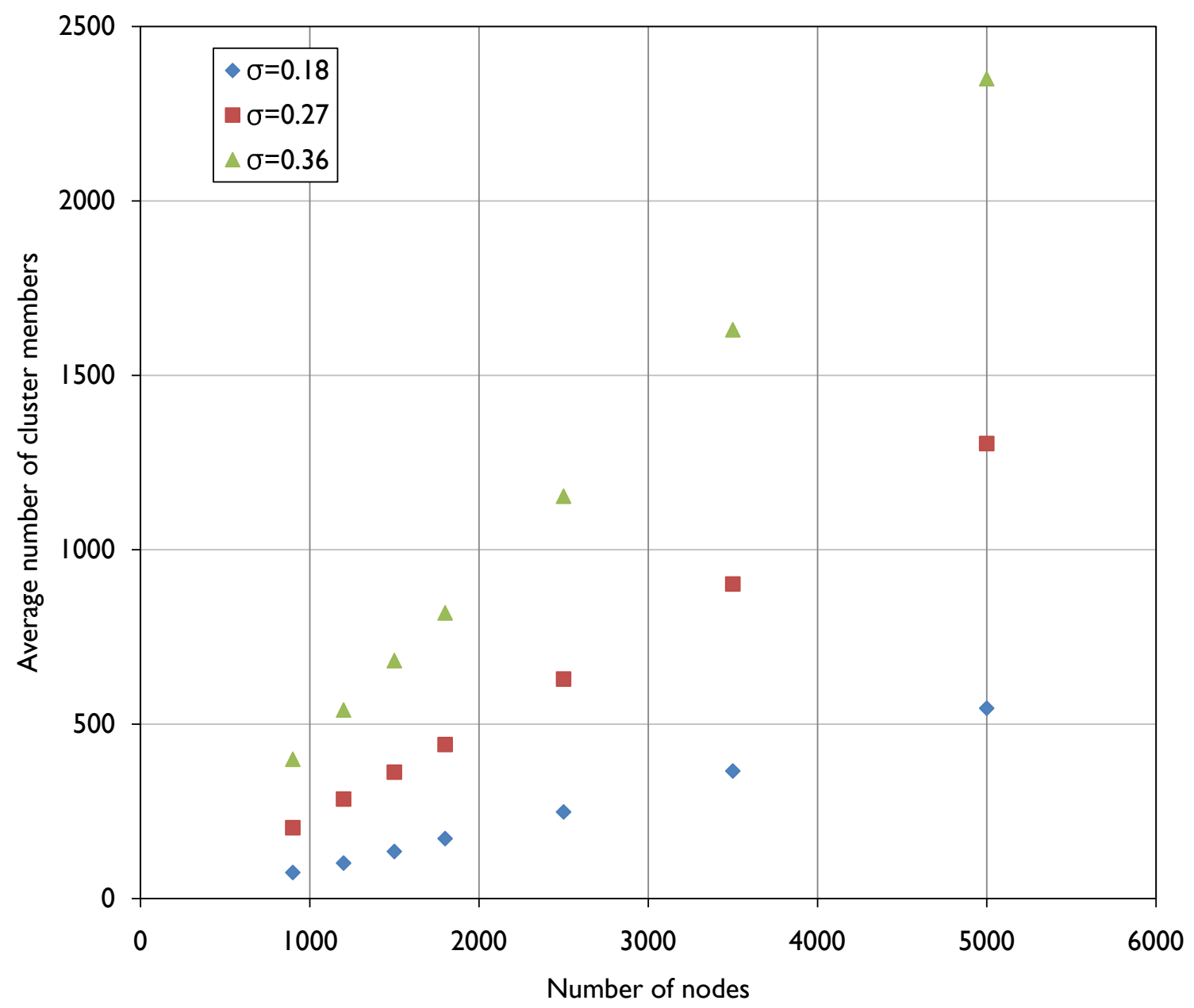

Fig. 14. Average number of cluster members for different $\sigma$.

\section{E. Summary}

In this chapter we first studied the proposed network control model over three aspects: the clustering control via the induction model, the node activation control over the inhibition model, and the clusterhead election over a modified inhibition model. Various stability and convergence issues were also discussed. We then conducted some asymptotic study over infinitely-large networks, where we gave an empirical estimate of the cluster size. 


\section{CHAPTER V}

\section{CONCLUSION}

In this thesis, inspired by inter-cell biological models, we first proposed a distributed clustering algorithm for WSNs based on the biological lateral induction model, and a subsequent algorithm for further node activation control based the biological lateral inhibition model. In addition, we proposed a clusterhead election algorithm based on a variant of the biological lateral inhibition model. Such a three-phase protocol is event-driven and runs in a purely distributed fashion. Specifically, when a target appears in the field, the sensor nodes create a compact cluster in the vicinity of the target by collaborating as a functional cluster. Then, via the competitive inhibition phase, only a subset of the cluster members remain active while the rest stay in idle mode to save energy. Finally, a clusterhead is elected among the active cluster members to collect and forward sensing reports.

Simulations for the induction algorithm on a network of finite size show that, by fostering collaborative interaction, the clustering phase of our model improves the energy efficiency through the construction of compact clusters. In addition, this procedure is purely distributed; all decisions by the nodes are performed locally by observing their neighboring environment. In the case of networks of infinite size, we examine the average number of cluster members.

\section{A. Future Work}

We can investigate a recovery model for large-scale wireless sensor networks in the premise of inter-cell signaling. Much like an organism heals from wounds, we would like the sensor nodes to respond to attacks in a way that will not compromise the network. A proposed solution is that nodes surrounding an affected area form a 
protective patch via local lateral induction, in a way reforming the cluster. Then, the nodes proceed with a subsequent phase of local lateral inhibition until some nodes around the affected region become active, and such active patterns could change periodically to save energy. 


\section{REFERENCES}

[1] I. F. Akyildiz, W. Su, Y. Sankarasubramaniam, and E. Cayirci, "Wireless sensor networks: A survey," Computer Networks, vol. 38, no. 4, pp. 393-422, March 2002.

[2] K. Martinez, J. K. Hart, and R. Ong, "Environmental sensor networks," Computer, vol. 37, no. 8, pp. 50-56, August 2004.

[3] A. Chandrakasan, R. Amirtharajah, S. Cho, J. Goodman, G. Konduri, J. Kulik, W. Rabiner, and A. Wang, "Design considerations for distributed micro-sensor systems," in Proc. of the IEEE 1999 Custom Integrated Circuits Conference, pp. 279-286, San Diego, CA, May 1999.

[4] R. Nelson, "ALERT Systems Organization," http://www.alertsystems.org.

[5] "CENS: Center for embedded networked sensing", http://research.cens.ucla.edu.

[6] J. Nemeroff, L. Garcia, D. Hampel, and S. DiPierro, "Networked sensor communications," in In Proc. of MILCOM, pp. 1462-1465, Anaheim, CA, October 2002.

[7] H. Baldus, K. Klabunde, and G. Muesch, "Reliable setup of medical body-sensor networks," in Proc. of EWSN, pp. 353-363, Berlin, Germany, Janurary 2004.

[8] T. T. Hsieh, "Using sensor networks for highway and traffic applications," Potentials, vol. 23, no. 2, pp. 13-16, April-May 2004.

[9] D. Estrin, R. Govinda, J. Heidemann, and S. Kumar, "Next centuary challenges: Scalable coordination in sensor networks," in Proc. of MobiCOM, pp. 263-270, Seattle, WA, August 1999. 
[10] C-Y. Chong and S. P. Kumar, "Sensor Networks: Evolution, Opportunities, and Challenges," in Proc. of the IEEE, vol. 91, no. 8, pp. 1247-1256, August 2003.

[11] C. Prehofer and C. Bettstetter, "Self-organization in communication networks: Principles and design paradigms," Communications Magazine, vol. 43, no. 7, pp. 78-85, July 2005.

[12] A. Arora, P. Dutta, S. Bapat, V. Kulathumani, H. Zhang, V. Naik, V. Mittal, H. Gao, M. Demirbas, M. Gouda, Y. Choi, T. Herman, S. Kulkarni, U. Arumugam, M. Nesterenko, A. Vora, and M. Miyashita, "A line in the sand: A wireless sensor network for target detection, classification, and tracking," Computer Networks, vol. 46, no. 5, pp. 605-634, December 2004.

[13] S. Cui, A. J. Goldsmith, and A. Bahai, "Energy efficiency of MIMO and cooperative MIMO in sensor networks," Selected Areas in Communications, vol. 22, no. 6, pp. 1089-1098, August 2004.

[14] X. Wang and S. Wang, "Collaborative signal processing for target tracking in distributed wireless sensor networks," Parallel and Distributed Computing, vol. 67, no. 5, pp. 501-515, May 2007.

[15] M. Chatterjee, S. K. Das, and D. Turgut, "WCA: A Weighted Clustering Algorithm for mobile ad hoc networks," Cluster Computing, vol. 5, no. 2, pp. 193-204, April 2002.

[16] W. R. Heinzelman, A. Chandrakasan, and H. Balakrishnan, "Energy-efficient communication protocol for wireless microsensor networks," in Proc. of the 33rd Hawaii International Conference on System Sciences, pp. 3005-3014, Maui, HI, January 2000. 
[17] G-Y. Jin, X-Y. Lu, and M-S. Park, "Dynamic clustering for object tracking in wireless sensor networks," Lecture Notes in Computer Science, vol. 4239, pp. 200-209, 2006.

[18] C-M. Liu, C-H. Lee, and L-C. Wang, "Distributed clustering algorithms for data-gathering in wireless mobile sensor networks," Parallel and Distributed Computing, vol. 67, no. 11, pp. 1187-1200, November 2007.

[19] M. Qin and R. Zimmermann. "VCA: An energy-efficient voting-based clustering algorithm for sensor networks," Universal Computer Science, 13(1):87-109, 2007.

[20] C. Sharp, S. Schaffert, A. Woo, N. Sastry, and C. Karlof, "Design and implementation of a sensor network system for vehicle tracking and autonomous interception," in Proc. of the Second European Workshop on Wireless Sensor Networks, pp. 93-107, Istanbul, Turkey, January 2005.

[21] X. Wang, J-J. Ma, S. Wang, and D-W. Bi, "Cluster-based dynamic energy management for collaborative target tracking in wireless sensor networks," Sensors, vol. 7, no. 7, pp. 1193-1215, July 2007.

[22] O. Younis and S. Fahmy, "HEED: A Hybrid, Energy-Efficient, Distributed clustering approach for ad hoc sensor networks," Mobile Computing, vol. 3, no. 4, pp. 366-379, October 2004.

[23] F. Zhao, J. Shin, and J. Reich, "Information-driven dynamic sensor collaboration," Signal Processing, vol. 19, no. 2, pp. 61-72, March 2002.

[24] C. Charalambous and S. Cui, "A bio-inspired distributed clustering algorithm for wireless sensor networks," in Proc. of the 4th Annual International Conference 
on Wireless Internet, Maui, HI, November 2008.

[25] A. Bailón-Plaza and M. C. H. van der Meulen, "A mathematical framework to study the effects of growth factor influences on fracture healing," Theoretical Biology, vol. 212, no. 2, pp. 191-209, September 2001.

[26] D. L. Nikolić, A. N. Boettiger, D. Bar-Sagi, J. D. Carbeck, and S. Y. Shvartsman, "Role of boundary conditions in an experimental model of epithelial wound healing," American Journal of Physiology-Cell Physiology, vol. 291, no. 1, pp. 68-75, July 2006 .

[27] N. Daudet and J. Lewis, "Two contrasting roles for notch activity in chick inner ear development: Specification of prosensory patches and lateral inhibition of hair-cell differentiation," Development, vol. 132, no. 3, pp. 541-551, February 2005.

[28] N. A. Monk, J. A. Sherratt, and M. R. Owen, "Spatiotemporal patterning in models of juxtacrine intercellular signalling with feedback," Institute for Mathematics and Its Applications, vol. 121, pp. 165-193, 2001.

[29] J. R. Collier, N. A. Monk, P. K. Maini, and J. H. Lewis, "Pattern formation by lateral inhibition with feedback: A mathematical model of delta-notch intercellular signalling," Theoretical Biology, vol. 183, no. 4, pp. 429-446, December 1996.

[30] M. R. Owen, J. A. Sherratt, and H. J. Wearing, "Lateral induction by juxtacrine signaling is a new mechanism for pattern formation," Developmental Biology, vol. 217, no. 1, pp. 54-61, January 2000. 
[31] E. Plahte, "Pattern formation in discrete cell lattices," Mathematical Biology, vol. 43, no. 5, pp. 411-445, November 2001.

[32] P. W. Sternberg, "Falling off the knife edge," Current Biology, vol. 3, no. 11, pp. 763-765, November 1993.

[33] M. R. Owen and J. A. Sherratt, "Mathematical modeling of juxtacrine cell signaling," Mathematical Biosciences, vol. 153, no. 2, pp. 125-150, November 1998.

[34] S. D. Webb and M. R. Owen, "Oscillations and patterns in spatially discrete models for developmental intercellular signalling," Mathematical Biology, vol. 48, no. 4, pp. 444-476, April 2004.

[35] E. Kreyszig, Advance Engineering Mathematics, New York, John Wiley \& Sons, Inc., 1999.

[36] J. B. Kruskal, "On the shortest spanning subtree of a graph and the traveling salesman problem," Proc. of the American Mathematical Society, vol. 7, no. 1, pp. 48-50, February 1956.

[37] W. Zhang and G. Cao, "DCTC: Dynamic Convoy Tree-based Collaboration for target tracking in sensor networks," Wireless Communications, vol. 3, no. 5, pp. 1689-1701, September 2004.

[38] M. Penrose, Random Geometric Graphs, Oxford Studies in Probability 5, Oxford University Press, 2003.

[39] P. Gupta, and P. R. Kumar, "Critical power for asymptotic connectivity in wireless networks," Stochastic Analysis, Control, Optimization and Applications A Volume in Honor of W.H. Fleming, pp. 547-566, 1998. 


\section{VITA}

Charalambos Charalambous was born in Nicosia, Cyprus. He received his Bachelor of Science in electrical engineering (with Minor in mathematics) from the Department of Electrical and Computer Engineering at The University of Arizona, Tucson, AZ, in May 2006. He received his Master of Science in electrical engineering from the Department of Electrical and Computer Engineering at Texas A\&M University, College Station, TX. His research topic was on biologically inspired modeling for wireless sensor networks, and he worked under the supervision of Dr. Shuguang Cui.

Mr. Charalambous may be reached via email at charalambos00@gmail.com, and by mail at: 214 Zachry Engineering Center, College Station, TX 77843-3128. 